\title{
AN ASYMPTOTIC MODEL BASED ON MATCHING FAR AND NEAR FIELD EXPANSIONS FOR THIN GRATINGS PROBLEMS
}

\author{
Peter B. Monk ${ }^{1}$, Cinthya Rivas ${ }^{2,3}$, Rodolfo Rodríguez ${ }^{2,3}$ And \\ MANUEl E. SOLANO ${ }^{2,3, *}$
}

\begin{abstract}
In this paper, we devise an asymptotic model for calculating electromagnetic diffraction and absorption in planar multilayered structures with a shallow surface-relief grating. Far from the grating, we assume that the solution can be written as a power series in terms of the grating thickness $\delta$, the coefficients of this expansion being smooth up to the grating. However, the expansion approximates the solution only sufficiently far from the grating (far field approximation). Near the grating, we assume that there exists another expansion in powers of $\delta$ (near field approximation). Moreover, there is an overlapping zone where both expansion are valid. The proposed model is based on matching the two expansions on this overlapping domain. Then, by truncating terms of order $\delta^{2}$ or higher, we obtain explicitly the equations satisfied by the lowest order terms in the power series. Under appropriate assumptions, we prove second order convergence of the error with respect to $\delta$. Finally, an alternative form, more convenient for implementation, is derived and discretized with finite elements to perform some numerical tests.
\end{abstract}

Mathematics Subject Classification. 65N30, 74M35.

Received October 16, 2019. Accepted July 28, 2020.

\section{INTRODUCTION}

An important class of light harvesting devices are three-dimensional structures composed by a multilayered material, usually a dielectric or a semiconductor, placed on top of a periodically corrugated metallic surface relief grating; for example, photovoltaic solar cells [25] or planar optical concentrators [24,26]. The electromagnetic field in these structures can be modeled by the frequency domain Maxwell's equations and it is of interest to optimize optical and geometrical parameters to maximize the light absorption [24,27]. In general, in this context, it is not possible to obtain closed-form expressions of the solution to Maxwell's equations. This is the reason why numerical methods play an important role in order to approximate the electromagnetic field. Amongst those that discretize the partial differential equations directly, probably the most popular are the finite-difference time-domain (FDTD) method [29], the rigorous coupled-wave approach (RCWA) $[4,12,14,15,20]$, the boundary

Keywords and phrases. diffraction grating, thin layers, asymptotic analysis, finite element method.

1 Department of Mathematical Sciences, University of Delaware, Newark, DE 19716, USA.

2 Departamento de Ingeniería Matemática, Facultad de Ciencias Físicas y Matemáticas, Universidad de Concepción, Casilla 160-C, Concepción, Chile.

3 Center for Research in Mathematical Engineering ( $\left.\mathrm{CI}^{2} \mathrm{MA}\right)$, Universidad de Concepción, Casilla 160-C, Concepción, Chile.

*Corresponding author: msolano@ing-mat-udec.cl, manolosolano@gmail.com 
integral equations method $[1,11]$ and the finite element method (FEM) $[21,27,28]$, the latter being preferred to simulate complicated structures.

These devices can exhibit extremely thin layers or very shallow grating corrugations compared to the total size of the structure. This feature affects the computational cost of mesh-based numerical solvers, since an extremely fine grid is needed to resolve the geometry. As an alternative, it is possible to devise an asymptotic model that approximates the electromagnetic field in the structure by replacing the thin layer by an interface where suitable transmission conditions are imposed [7-9, 19,22]. In this direction, an asymptotic model for calculating electromagnetic diffraction and absorption in planar multilayered structures with a shallow surfacerelief grating has been devised in [23]. The numerical results reported in this reference show that when the asymptotic expansion of the solution is truncated to second order terms, third-order convergence with respect to the thickness $\delta$ of the thin grating layer is obtained for transverse electric polarization (s-polarization), and at least second-order convergence for the transverse magnetic polarization ( $p$-polarization). However, there is no rigorous mathematical framework supporting this approach. Actually, to the best of the authors' knowledge, there is no asymptotic model for this problem for which error estimates have been rigorously proved.

In this paper, we describe an alternative asymptotic model inspired by that in reference [8], for which we succeed in deriving an error analysis. Far from the grating, we again assume that the solution can be written as a power series in terms of $\delta$, the coefficients of this expansion being smooth up to the grating. This expansion is a far field approximation, in the sense that it approximates the solution only sufficiently far from the grating. Near the grating, we assume that there exists another expansion in powers of $\delta$, which is a near field approximation. Moreover, there is an overlapping zone where both expansions hold. The asymptotic model is based on matching these two expansions on the overlapping domain. We truncate the expansion of the solution to first order terms and, under appropriate assumptions, prove convergence of the error with order $\delta^{2}$.

The rest of the paper is organized as follows. In Section 2 we describe the boundary-value problem. In Section 3 we formulate the asymptotic model. In Section 4 we prove error estimates for the asymptotic expansion of the solution. In Section 5 we introduce an alternative formulation more convenient for the implementation of a finite element discretization. In Section 6 we report some numerical tests. Finally, we include in an appendix the derivation of the approximate transmission conditions and other auxiliary results.

\section{MODEL PROBLEM}

First, let us fix some notation. The free-space wavenumber, wavelength and intrinsic impedance are respectively denoted by $k_{0}:=\omega \sqrt{\varepsilon_{0} \mu_{0}}, \lambda_{0}:=2 \pi / k_{0}$ and $\eta_{0}:=\sqrt{\mu_{0} / \varepsilon_{0}}$, where $\mu_{0}>0$ and $\varepsilon_{0}>0$ are respectively the magnetic permeability and the electric permittivity of free space and $\omega>0$ is the angular frequency. For a particular material, let $\varepsilon_{r}:=\varepsilon / \varepsilon_{0}$ be the relative electric permittivity (with $\varepsilon$ being the absolute permittivity), which in general is a complex-valued function.

Consider a simplified structure motivated by solar cell applications that occupies the region

$$
\Phi:=\left\{(x, y, z) \in \mathbb{R}^{3}:|x|<\infty,|y|<\infty,-L_{m}<z<L_{d}\right\} .
$$

Within this region, the relative permittivity is a periodic function of $x \in(-\infty, \infty)$ with period $L$, that also varies with $z \in\left(-L_{m}, L_{d}\right)$ but not with $y \in(-\infty, \infty)$; namely, $\varepsilon_{r}(x, z)=\varepsilon_{r}(x+m L, z) \forall m \in \mathbb{Z}$. The half spaces $z<-L_{m}$ and $z>L_{d}$ are occupied by air, so that the relative permittivity therein is $\varepsilon_{r}(x, z) \equiv 1$.

The wave propagation in the solar cell is governed by the time-harmonic Maxwell equations:

$$
\left\{\begin{array}{l}
\nabla \times \boldsymbol{E}=i \omega \mu_{0} \boldsymbol{H} \\
\nabla \times \boldsymbol{H}=-i \omega \varepsilon_{0} \varepsilon_{r}(x, z) \boldsymbol{E},
\end{array}\right.
$$

where $\boldsymbol{E}$ and $\boldsymbol{H}$ denote the electric and magnetic fields, respectively.

The upper boundary of the solar-cell $\left(z=L_{d}\right)$ is illuminated by an obliquely incident plane wave whose electric field is given by

$$
\boldsymbol{E}_{\text {inc }}(x, y, z)=\left\{a_{s} \hat{\boldsymbol{u}}_{y}+a_{p}\left(\hat{\boldsymbol{u}}_{x} \cos \theta+\hat{\boldsymbol{u}}_{z} \sin \theta\right)\right\} \exp \left\{i k_{0}\left(x \sin \theta-\left(z-L_{d}\right) \cos \theta\right)\right\}, \quad z \geq L_{d},
$$




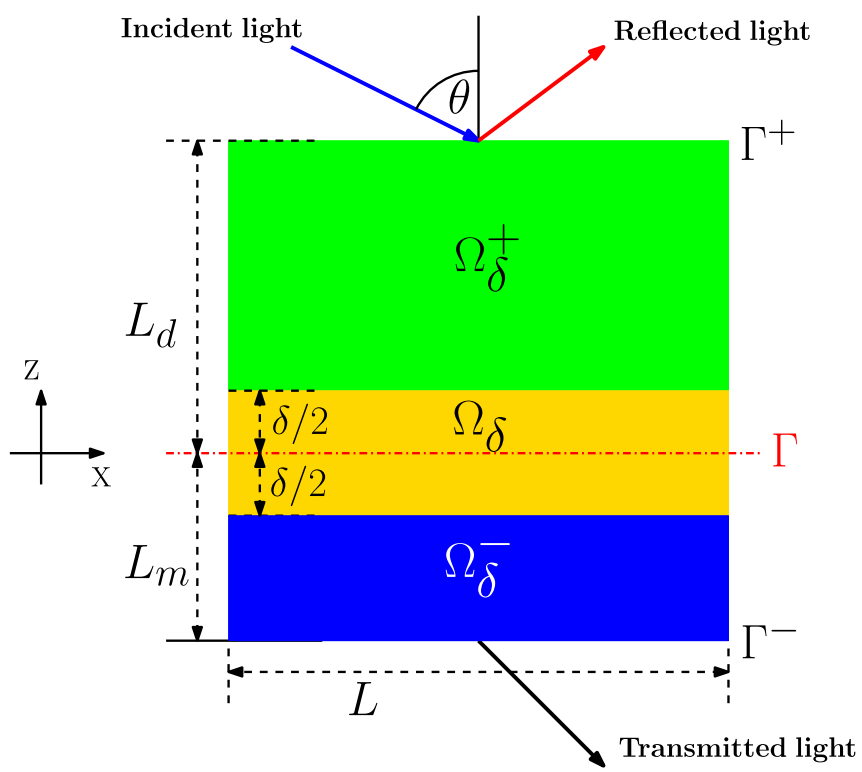

FIGURE 1. Domain $\Omega$ showing the geometric subdomains and notation.

where $\hat{\boldsymbol{u}}_{x}, \hat{\boldsymbol{u}}_{y}$ and $\hat{\boldsymbol{u}}_{z}$ are the Cartesian unit vectors, $\theta$ is the angle of incidence with respect to the $z$ axis, and $a_{s}$ and $a_{p}$ are data coefficients. This quantity, $\boldsymbol{E}_{\text {inc }}$, is the only source term of the problem. From (2.1a), the corresponding incident magnetic field is given by

$$
\boldsymbol{H}_{\mathrm{inc}}=\frac{1}{i \omega \mu_{0}} \nabla \times \boldsymbol{E}_{\mathrm{inc}}, \quad z \geq L_{d} .
$$

Note that $\left(\boldsymbol{E}_{\text {inc }}, \boldsymbol{H}_{\text {inc }}\right)$ satisfies $(2.1 \mathrm{a})$ when $\varepsilon_{r}=1$.

Since all the quantities do not depend on $y$, these equations can be written in any section for $y$ fixed. Moreover, because of the periodic character of the physical coefficients, the problem can be posed over one period, $0<x<L$, by imposing appropriate quasi-periodic conditions that will be specified below $(c f .(2.5))$. Therefore, we restrict the domain of our problem to the so called unit cell:

$$
\Omega:=\left\{(x, z) \in \mathbb{R}^{2}: 0<x<L,-L_{m}<z<L_{d}\right\} .
$$

Moreover, we introduce the following notation (see Fig. 1):

$$
\begin{aligned}
\Gamma^{+} & :=\left\{(x, z) \in \Omega: z=L_{d}\right\}, \\
\Gamma & :=\{(x, z) \in \Omega: z=0\}, \\
\Gamma^{-} & :=\left\{(x, z) \in \Omega: z=-L_{m}\right\}, \\
\Omega_{\delta}^{+} & :=\left\{(x, z) \in \Omega: \frac{\delta}{2}<z<L_{d}\right\}, \\
\Omega_{\delta} & :=\left\{(x, z) \in \Omega:-\frac{\delta}{2}<z<\frac{\delta}{2}\right\}, \\
\Omega_{\delta}^{-} & :=\left\{(x, z) \in \Omega:-L_{m}<z<-\frac{\delta}{2}\right\} .
\end{aligned}
$$

The region $\Omega_{\delta}^{+}$is occupied by an isotropic homogeneous dielectric material of real relative permittivity $\varepsilon_{r}^{+}$. The region $\Omega_{\delta}^{-}$is occupied by a homogeneous metal of complex relative permittivity $\varepsilon_{r}^{-}$. In the middle region $\Omega_{\delta}$, which is occupied by the grating, we assume that the complex relative permittivity $\varepsilon_{r}^{g}$ varies only with $x$. 
Therefore, the relative permittivity of the entire unit cell is given by

$$
\varepsilon_{r}(x, z):= \begin{cases}\varepsilon_{r}^{+}, & (x, z) \in \Omega_{\delta}^{+}, \\ \varepsilon_{r}^{g}(x), & (x, z) \in \Omega_{\delta} \\ \varepsilon_{r}^{-}, & (x, z) \in \Omega_{\delta}^{-} .\end{cases}
$$

All the permittivities are assumed not to vanish. Moreover we assume that $\varepsilon_{r}^{g}$ is infinitely differentiable in $[0, L]$ and the following inequalities, which are typically fulfilled in the applications:

$$
\left\{\begin{array}{l}
\varepsilon_{r}^{+}>0, \\
\operatorname{Im}\left(\varepsilon_{r}^{-}\right)>0 \quad \text { or } \quad \operatorname{Re}\left(\varepsilon_{r}^{-}\right)>0 \quad \text { when } \operatorname{Im}\left(\varepsilon_{r}^{-}\right)=0, \\
\operatorname{Im}\left(\varepsilon_{r}^{g}(x)\right)>0 \quad \forall x \in[0, L] \quad \text { or } \quad \operatorname{Re}\left(\varepsilon_{r}^{g}(x)\right)>0 \quad \forall x \in[0, L], \quad \text { when } \quad \operatorname{Im}\left(\varepsilon_{r}^{g}\right) \equiv 0 .
\end{array}\right.
$$

\subsection{Scalar equations and boundary conditions}

Problem (2.1) can be decoupled into two separate problems with corresponding coefficients $a_{s}$ and $a_{p}$ in the source term (2.1b). The respective decoupled problems are called the $s$ - and $p$-polarization states. Both reduce to the following common form of the Helmholtz equation:

$$
\nabla \cdot(B(x, z) \nabla u(x, z))+k_{0}^{2} b(x, z) u(x, z)=0, \quad(x, z) \in \Omega,
$$

where, for the $s$-polarization state,

$$
u(x, z)=E_{y}(x, z), \quad B(x, z)=1, \quad b(x, z)=\varepsilon_{r}(x, z),
$$

and for the $p$-polarization state,

$$
u(x, z)=-\eta_{0} H_{y}(x, z), \quad B(x, z)=\frac{1}{\varepsilon_{r}(x, z)}, \quad b(x, z)=1 .
$$

The data of each of these problems are the corresponding components of the incident plane waves (2.1b) and (2.1c):

$$
u_{\text {inc }}(x, z)= \begin{cases}a_{s} \exp \left(i k_{0}\left(x \sin \theta-\left(z-L_{d}\right) \cos \theta\right)\right), & \text { for the } s \text {-polarization } \\ a_{p} \exp \left(i k_{0}\left(x \sin \theta-\left(z-L_{d}\right) \cos \theta\right)\right), & \text { for the } p \text {-polarization }\end{cases}
$$

The total field $u$ satisfies the following relations:

$$
\begin{cases}B(x, z) \frac{\partial u}{\partial z}(x, z)=\left(T^{-} u\right)(x, z) & \text { on } \Gamma^{-}, \\ B(x, z)\left(\frac{\partial u}{\partial z}(x, z)-\frac{\partial u_{\text {inc }}}{\partial z}(x, z)\right)=\left(T^{+} u\right)(x, z)-\left(T^{+} u_{\text {inc }}\right)(x, z) & \text { on } \Gamma^{+},\end{cases}
$$

where $T^{-}$and $T^{+}$are the corresponding Dirichlet-to-Neumann operators (see [5]).

In addition, $u(x, z)$ satisfies the quasi-periodicity conditions

$$
\left.\begin{array}{l}
u(L, z)=\exp (i \alpha L) u(0, z), \\
\frac{\partial u}{\partial x}(L, z)=\exp (i \alpha L) \frac{\partial u}{\partial x}(0, z),
\end{array}\right\} \quad z \in\left(-L_{m}, L_{d}\right)
$$

where $\alpha:=k_{0} \sin \theta$. 
Altogether, we arrive at the following problem:

$$
\begin{cases}\nabla \cdot(B(x, z) \nabla u(x, z))+k_{0}^{2} b(x, z) u(x, z)=0 & \text { in } \Omega, \\ u(L, z)=e^{i \alpha L} u(0, z), & z \in\left(-L_{m}, L_{d}\right), \\ \frac{\partial u}{\partial x}(L, z)=e^{i \alpha L} \frac{\partial u}{\partial x}(0, z), & z \in\left(-L_{m}, L_{d}\right), \\ B(x, z) \frac{\partial u}{\partial z}(x, z)=\left(T^{-} u\right)(x, z) & \text { on } \Gamma^{-}, \\ B(x, z)\left(\frac{\partial u}{\partial z}(x, z)-\frac{\partial u_{\text {inc }}}{\partial z}(x, z)\right)=\left(T^{+} u\right)(x, z)-\left(T^{+} u_{\text {inc }}\right)(x, z) & \text { on } \Gamma^{+} .\end{cases}
$$

The next step is to write a variational formulation of this problem. To this end we define

$$
H_{\alpha}^{1}(\Omega):=\left\{v \in H^{1}(\Omega): v(L, z)=e^{i \alpha L} v(0, z) \quad \forall z \in\left(-L_{m}, L_{d}\right)\right\} .
$$

Testing the first equation in (2.6) with $v \in H_{\alpha}^{1}(\Omega)$ and integrating by parts lead to the following problem: Find $u \in H_{\alpha}^{1}(\Omega)$ such that

$$
a(u, v)=L(v) \quad \forall v \in H_{\alpha}^{1}(\Omega)
$$

where

$$
\left\{\begin{array}{l}
a(w, v):=\int_{\Omega}\left(B \nabla w \cdot \nabla \bar{v}-k_{0}^{2} b w \bar{v}\right) \mathrm{d} x \mathrm{~d} z-\int_{\Gamma^{+}}\left(T^{+} w\right) \bar{v} \mathrm{~d} x-\int_{\Gamma^{-}}\left(T^{-} w\right) \bar{v} \mathrm{~d} x, \quad v, w \in H_{\alpha}^{1}(\Omega), \\
L(v):=\int_{\Gamma^{+}}\left(B \frac{\partial u_{\mathrm{inc}}}{\partial z}-T^{+} u_{\text {inc }}\right) \bar{v} \mathrm{~d} x, \quad v \in H_{\alpha}^{1}(\Omega) .
\end{array}\right.
$$

Assumption 2.1. We assume that problem (2.7) is well posed for all but at most a sequence of countable frequencies $\omega_{j}$ with $\left|\omega_{j}\right| \rightarrow+\infty$ and we restrict our attention to $\omega \neq \omega_{j}$. For each $\omega \neq \omega_{j}$, there exists a constant $C>0$ such that

$$
\|w\|_{H^{1}(\Omega)} \leq C \sup _{\substack{v \in H_{\alpha}^{1}(\Omega) \\ v \neq 0}} \frac{|a(w, v)|}{\|v\|_{H^{1}(\Omega)}} \quad \forall w \in H_{\alpha}^{1}(\Omega) .
$$

Moreover, we assume that $C$ is independent of $\delta$.

Remark 2.2. The first part of this assumption has been proved in Theorem 3.3 of [10], in case that $\varepsilon_{r}^{g}(x)$ is piecewise constant and inequalities (2.2) hold true. The assumption that $C$ is independent of $\delta$ can be proved, for example, if the grating is non-trapping [6]. In addition, by following the arguments in Theorem 2.1 of [13], it is possible to show that $C$ is independent of $\delta$ when $\delta$ is small enough. On the other hand, this assumption implicitly avoids the presence of resonances due to Rayleigh-Wood anomalies [18].

\section{Asymptotic model}

In this section, we will introduce an alternative to deal with the thin grating layer $\Omega_{\delta}$. In fact, problem (2.6) constitutes the full model, which will be approximated by an asymptotic model (valid in the limit $\delta \rightarrow 0$ ), where the effect of the grating layer $\Omega_{\delta}$ will be taken into account by means of appropriate approximate transmission conditions across $\Gamma$.

With this aim, we consider an auxiliary problem based on another partition of the domain $\Omega$ into subdomains $\Omega^{+}$and $\Omega^{-}$, where (see Fig. 2)

$$
\Omega^{+}:=(0, L) \times\left(0, L_{d}\right) \quad \text { and } \quad \Omega^{-}:=(0, L) \times\left(-L_{m}, 0\right),
$$




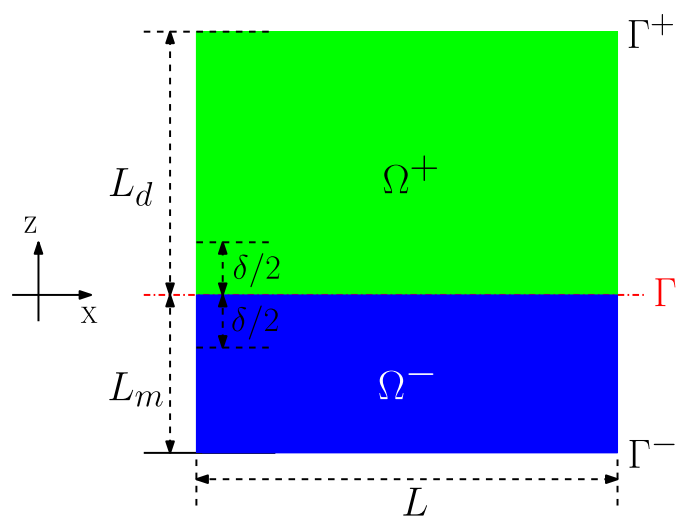

FIGURE 2. Decomposition of the domain $\Omega$ with the thin interface removed.

with relative permittivity

$$
\varepsilon_{r}(x, z):=\left\{\begin{array}{l}
\varepsilon_{r}^{+},(x, z) \in \Omega^{+} \\
\varepsilon_{r}^{-},(x, z) \in \Omega^{-}
\end{array}\right.
$$

For any function $v: \Omega^{+} \cup \Omega^{-} \longrightarrow \mathbb{C}$, we denote $v^{+}:=\left.v\right|_{\Omega^{+}}$and $v^{-}:=\left.v\right|_{\Omega^{-}}$. In general, we identify $v$ with the pair of functions $\left(v^{+}, v^{-}\right)$. Note that in this asymptotic model, $B^{ \pm}$and $b^{ \pm}$as defined in (2.3) and (2.4) are constant.

Inspired by the procedure used in [8], we consider two different expansions of the solution $u(x, z)$ : one in the far field zone $(|z| \gg \delta / 2)$ and the other in the near field zone $(|z| \sim \delta / 2)$. In what follows, we describe each of them in detail.

\subsection{Far field equations}

In the far field, we make the following assumption.

Assumption 3.1. Outside the grating, we assume that $u$ can be expanded in a standard series in powers of $\delta$ :

$$
u(x, z)= \begin{cases}\sum_{n=0}^{\infty} \delta^{n} u_{n}^{+}(x, z), & z \geq \delta / 2, \\ \sum_{n=0}^{\infty} \delta^{n} u_{n}^{-}(x, z), & z \leq-\delta / 2,\end{cases}
$$

where the far field terms $u_{n}^{ \pm}$defined in $\Omega^{ \pm}$are quasi-periodic in $x$ and infinitely smooth up to $\Gamma$. Moreover, we assume that each $u_{n}^{ \pm}$can be expanded in a power series with respect to the $z$-coordinate around zero, i.e.,

$$
u_{n}^{ \pm}(x, z)=\sum_{k=0}^{\infty} \frac{z^{k}}{k !} \frac{\partial^{k} u_{n}^{ \pm}}{\partial z^{k}}(x, 0), \quad(x, z) \in \Omega^{ \pm}, \quad n \geq 0 .
$$


To derive the equations satisfied by the far field terms $u_{n}^{ \pm}$, we insert the asymptotic expansion (3.1) into (2.6) and extend the first equation to the whole $\Omega^{ \pm}$. Then, equating the terms with the same powers of $\delta$ we obtain:

$$
\begin{cases}\nabla \cdot\left(B^{ \pm} \nabla u_{n}^{ \pm}(x, z)\right)+k_{0}^{2} b^{ \pm} u_{n}^{ \pm}(x, z)=0 & \text { in } \Omega^{ \pm}, \\ u_{n}^{ \pm}(L, z)=e^{i \alpha L} u_{n}^{ \pm}(0, z), & z \in\left(-L_{m}, L_{d}\right), \\ \frac{\partial u_{n}^{ \pm}}{\partial x}(L, z)=e^{i \alpha L} \frac{\partial u_{n}^{ \pm}}{\partial x}(L, z), & z \in\left(-L_{m}, L_{d}\right), \\ B^{-} \frac{\partial u_{n}^{-}}{\partial z}(x, z)=\left(T^{-} u_{n}^{-}\right)(x, z) & \text { on } \Gamma^{-}, n \geq 0, \\ B^{+} \frac{\partial u_{n}^{+}}{\partial z}(x, z)=\left(T^{+} u_{n}^{+}\right)(x, z) & \text { on } \Gamma^{+}, n \geq 1, \\ B^{+} \frac{\partial u_{0}^{+}}{\partial z}(x, z)-B^{+} \frac{\partial u_{\text {inc }}}{\partial z}(x, z)=\left(T^{+} u_{0}^{+}\right)(x, z)-\left(T^{+} u_{\text {inc }}\right)(x, z) & \text { on } \Gamma^{+} .\end{cases}
$$

Note that to determine $u_{n}^{ \pm}$entirely, we need to prescribe transmission conditions relating $u_{n}^{+}$and $u_{n}^{-}$on $\Gamma$.

\subsection{Near field equations}

In the near field $(|z|<2 \delta)$ we resort to an asymptotic expansion based on the original partition of the domain $\Omega$ into the subdomains $\Omega_{\delta}^{+}, \Omega_{\delta}$ and $\Omega_{\delta}^{-}$(see Fig. 1). With this aim, we rescale the solution $u$ of problem (2.7) with respect to the thickness of the grating layer by changing the variable $z$ to $\xi:=\frac{z}{\delta}$ and make the following assumption.

Assumption 3.2. Near the grating, we assume that there exists an expansion, which, after rescaling by $\delta$, can be written as follows:

$$
u(x, z)=\sum_{n=0}^{\infty} \delta^{n} U_{n}\left(x, \frac{z}{\delta}\right), \quad|z| \leq 2 \delta,
$$

where $U_{n}(x, \xi)$ are quasi-periodic functions in $x$ and continuous in $(0, L) \times(-2,2)$ with $B(x, \delta \xi) \frac{\partial U_{n}}{\partial \xi}(x, \xi)$ also continuous. Furthermore, we assume that $U_{n}$ are infinitely smooth out of the grating, that is, when $\frac{1}{2} \leq|\xi| \leq 2$.

The first assumption is needed to ensure that the series in the identification of the matching conditions are convergent. On the other hand, it is natural to assume the quasi-periodicity condition, since we expect the series to converge to a quasi-periodic function. The continuity assumptions for $U_{n}$ and $B \partial_{\xi} U_{n}$ are required to derive the transmission conditions (see Appendix A). Finally, the last assumption will be employed to deduce the matching conditions and also to obtain the error estimates.

With any function $U(x, \xi)$, we associate the function

$$
U^{\delta}(x, z):=U\left(x, \frac{z}{\delta}\right)
$$

and recall the chain rule:

$$
\frac{\partial U^{\delta}}{\partial z}=\frac{1}{\delta} \frac{\partial U}{\partial \xi}
$$

Therefore, the expansion (3.3) can be rewritten as

$$
u(x, z)=\sum_{n=0}^{\infty} \delta^{n} U_{n}^{\delta}(x, z) .
$$


To derive the equations satisfied by the near field terms $U_{n}$, we insert this asymptotic expansion into (2.6). Then, from the first equation and the assumption that $U_{n}$ are smooth, we have that

$$
\sum_{n=0}^{\infty} \delta^{n}\left\{\nabla \cdot\left(B \nabla U_{n}^{\delta}\right)+k_{0}^{2} b U_{n}^{\delta}\right\}=0 .
$$

Now, using the chain rule and denoting $\xi:=\frac{z}{\delta}$, we obtain

$$
\begin{aligned}
& \nabla \cdot\left(B(x, z) \nabla U_{n}^{\delta}(x, z)\right)+k_{0}^{2} b(x, z) U_{n}^{\delta}(x, z) \\
& \quad=\frac{1}{\delta^{2}} \frac{\partial}{\partial \xi}\left(B(x, \delta \xi) \frac{\partial U_{n}(x, \xi)}{\partial \xi}\right)+\frac{\partial}{\partial x}\left(B(x, \delta \xi) \frac{\partial U_{n}(x, \xi)}{\partial x}\right)+k_{0}^{2} b(x, \delta \xi) U_{n}(x, \xi) .
\end{aligned}
$$

Hence, equating the terms with the same power of $\delta$ and using the convention that $U_{\ell}^{\delta}=0$ for $\ell<0$, we obtain the following equations for the near field terms $U_{n}, n \geq 0$ :

$$
\left\{\begin{array}{lr}
\frac{\partial}{\partial \xi}\left(B(x, \delta \xi) \frac{\partial U_{n}(x, \xi)}{\partial \xi}\right)=-\frac{\partial}{\partial x}\left(B(x, \delta \xi) \frac{\partial U_{n-2}(x, \xi)}{\partial x}\right)-k_{0}^{2} b(x, \delta \xi) U_{n-2}(x, \xi),(x, \xi) \in(0, L) \times(-2,2), \\
U_{n}(L, \xi)=e^{i \alpha L} U_{n}(0, \xi), & \xi \in(-2,2), \\
\frac{\partial U_{n}}{\partial x}(L, \xi)=e^{i \alpha L} \frac{\partial U_{n}}{\partial x}(0, \xi), & \xi \in(-2,2) .
\end{array}\right.
$$

\subsection{Matching conditions}

To determine the terms $u_{n}^{-}, u_{n}^{+}$and $U_{n}$, we need additional matching conditions that will be obtained from the fact that the far and near field expansions have to coincide on certain overlapping zones $C_{\delta}^{ \pm}$. These zones should be disjoint with the grating layer but they should approach the interface $\Gamma$ as $\delta$ goes to zero. Because of this, we define the following overlapping domain, where expansions (3.1) and (3.3) are both valid:

$$
C_{\delta}:=C_{\delta}^{+} \cup C_{\delta}^{-} \quad \text { with } \quad C_{\delta}^{+}:=(0, L) \times(\delta, 2 \delta) \quad \text { and } \quad C_{\delta}^{-}:=(0, L) \times(-2 \delta,-\delta) .
$$

From Assumption 3.1, outside the grating (and so in particular in $C_{\delta}$ ), we have that

$$
u(x, z)=\sum_{n=0}^{\infty} \sum_{k=0}^{\infty} \delta^{n} \frac{z^{k}}{k !} \frac{\partial^{k} u_{n}^{ \pm}}{\partial z^{k}}(x, 0) .
$$

In turn, for the near field expansion, we have the following result regarding the behavior of the terms $U_{n}$ in the overlapping areas.

Proposition 3.3. There exist infinitely smooth quasi-periodic functions $p_{n, k}^{ \pm}$such that

$$
U_{n}(x, \xi)=\sum_{k=0}^{n+1} p_{n, k}^{ \pm}(x) \xi^{k}, \quad x \in(0, L), \quad \frac{1}{2} \leq|\xi| \leq 2 .
$$

Let us remark that the equation (3.7) is an abbreviated form of

$$
U_{n}(x, \xi)=\left\{\begin{array}{lll}
\sum_{k=0}^{n+1} p_{n, k}^{+}(x) \xi^{k}, & x \in(0, L), & \frac{1}{2} \leq \xi \leq 2, \\
\sum_{k=0}^{n+1} p_{n, k}^{-}(x) \xi^{k}, & x \in(0, L), & -2 \leq \xi \leq-\frac{1}{2} .
\end{array}\right.
$$

Similar notation will be used for other quantities in what follows. 
Proof. We proceed as in [8] in the simpler framework of our problem. For what follows, recall that the coefficients $B^{ \pm}(x, \delta \xi)$ and $b^{ \pm}(x, \delta \xi)$ are constant in $\Omega^{ \pm}$.

- For $n=0$, the first equation in (3.5) reduces to

$$
\frac{\partial}{\partial \xi}\left(B^{ \pm} \frac{\partial U_{0}(x, \xi)}{\partial \xi}\right)=0
$$

Then, there exist functions $p_{0,0}^{ \pm}(x)$ and $p_{0,1}^{ \pm}(x)$ such that

$$
U_{0}(x, \xi)=p_{0,0}^{ \pm}(x)+p_{0,1}^{ \pm}(x) \xi, \quad x \in(0, L), \quad \frac{1}{2} \leq|\xi| \leq 2 .
$$

Moreover, since we have assumed that $U_{n}$ is infinitely smooth for $\frac{1}{2} \leq|\xi| \leq 2$, we derive that $p_{0,0}^{ \pm}(x)$ and $p_{0,1}^{ \pm}(x)$ are infinitely smooth, too.

- For $n=1$, the first equation in (3.5) also reduces to

$$
\frac{\partial}{\partial \xi}\left(B^{ \pm} \frac{\partial U_{1}(x, \xi)}{\partial \xi}\right)=0
$$

Then, as above, there exist smooth functions $p_{1,0}^{ \pm}(x)$ and $p_{1,1}^{ \pm}(x)$ such that

$$
U_{1}(x, \xi)=p_{1,0}^{ \pm}(x)+p_{1,1}^{ \pm}(x) \xi, \quad x \in(0, L), \quad \frac{1}{2} \leq|\xi| \leq 2 .
$$

- For $n=2$, the first equation in (3.5) reduces to

$$
\begin{aligned}
\frac{\partial}{\partial \xi}\left(B^{ \pm} \frac{\partial U_{2}(x, \xi)}{\partial \xi}\right) & =-\left\{\frac{\partial}{\partial x}\left(B^{ \pm} \frac{\partial U_{0}(x, \xi)}{\partial x}\right)+k_{0}^{2} b^{ \pm} U_{0}(x, \xi)\right\} \\
& =-\left\{\left(B^{ \pm} \frac{\partial^{2} p_{0,0}^{ \pm}(x)}{\partial x^{2}}+k_{0}^{2} b^{ \pm} p_{0,0}^{ \pm}(x)\right)+\left(B^{ \pm} \frac{\partial^{2} p_{0,1}^{ \pm}(x)}{\partial x^{2}}+k_{0}^{2} b^{ \pm} p_{0,1}^{ \pm}(x)\right) \xi\right\} .
\end{aligned}
$$

Then, once more, there exist smooth functions $p_{2,0}^{ \pm}(x), p_{2,1}^{ \pm}(x), p_{2,2}^{ \pm}(x)$ and $p_{2,3}^{ \pm}(x)$ such that

$$
U_{2}(x, \xi)=p_{2,0}^{ \pm}(x)+p_{2,1}^{ \pm}(x) \xi+p_{2,2}^{ \pm}(x) \xi^{2}+p_{2,3}^{ \pm}(x) \xi^{3}, \quad x \in(0, L), \quad \frac{1}{2} \leq|\xi| \leq 2 .
$$

- An induction argument allows us to show that the proposition holds for all $n \geq 0$.

Now, we are in a position to settle matching conditions between both expansions, which is done in the following proposition. We emphasize that the identifications made in its proof are only formal, since the (pointwise) convergence of the series is not proved but assumed (cf. Assumption 3.2).

Proposition 3.4. For $n \geq 0$ and $0 \leq k \leq n$,

$$
U_{n}(x, \xi)=\sum_{k=0}^{n} \frac{\xi^{k}}{k !} \frac{\partial^{k} u_{n-k}^{ \pm}}{\partial z^{k}}(x, 0), \quad x \in(0, L), \quad \frac{1}{2} \leq|\xi| \leq 2 .
$$

Proof. By substituting (3.7) into the near field expansion (3.3), we obtain

$$
u(x, z)=\sum_{n=0}^{\infty} \sum_{k=0}^{n+1} \delta^{n} p_{n, k}^{ \pm}(x)\left(\frac{z}{\delta}\right)^{k}=\sum_{n=0}^{\infty} \sum_{k=0}^{n+1} \delta^{n-k} p_{n, k}^{ \pm}(x) z^{k}, \quad x \in(0, L), \quad \frac{\delta}{2} \leq|z| \leq 2 \delta .
$$


The identification on the overlapping zones of the far field (3.6) with this expression for the near field leads to

$$
\sum_{n=0}^{\infty} \sum_{k=0}^{\infty} \delta^{n} \frac{z^{k}}{k !} \frac{\partial^{k} u_{n}^{ \pm}}{\partial z^{k}}(x, 0)=\sum_{n=0}^{\infty} \sum_{k=0}^{n+1} \delta^{n-k} p_{n, k}^{ \pm}(x) z^{k}=\sum_{k=0}^{\infty} \sum_{\substack{n=k-1, n \geq 0}}^{\infty} \delta^{n-k} p_{n, k}^{ \pm}(x) z^{k}=\sum_{k=0}^{\infty} \sum_{\substack{n=-1, n+k \geq 0}}^{\infty} \delta^{n} p_{n+k, k}^{ \pm}(x) z^{k}
$$

for all $(x, z) \in C_{\delta}$. Then, exchanging the order of summation,

$$
\sum_{n=0}^{\infty} \sum_{k=0}^{\infty} \delta^{n} \frac{z^{k}}{k !} \frac{\partial^{k} u_{n}^{ \pm}}{\partial z^{k}}(x, 0)=\sum_{n=-1}^{\infty} \sum_{\substack{k=0 \\ n+k}}^{\infty} \delta^{n} z^{k} p_{n+k, k}^{ \pm}(x), \quad(x, z) \in C_{\delta} .
$$

Identifying the terms with the same power of $\delta$, we arrive at

$$
p_{n, k}^{ \pm}(x)= \begin{cases}0, & \text { if } \quad k=n+1 \\ \frac{1}{k !} \frac{\partial^{k} u_{n-k}^{ \pm}}{\partial z^{k}}(x, 0), & \text { if } \quad 0 \leq k \leq n\end{cases}
$$

for all $n \geq 0$, which substituted into (3.7) allows us to conclude the proof.

\subsection{Truncated asymptotic expansion}

Outside the grating, we approximate $u$ by

$$
u(x, z) \approx u_{0}^{ \pm}(x, z)+\delta u_{1}^{ \pm}(x, z), \quad|z| \geq \frac{\delta}{2},
$$

and inside the grating by

$$
u(x, z) \approx U_{0}\left(x, \frac{z}{\delta}\right)+\delta U_{1}\left(x, \frac{z}{\delta}\right), \quad|z|<\frac{\delta}{2} .
$$

Given $v^{ \pm}$defined in $\Omega^{ \pm}$we use the following notation for its jump and average on $\Gamma$ (i.e., at $z=0$ ):

$$
\begin{aligned}
& {[v]:=v^{+}(x, 0)-v^{-}(x, 0),} \\
& \langle v\rangle:=\frac{v^{+}(x, 0)+v^{-}(x, 0)}{2} .
\end{aligned}
$$

Also, we denote the coefficients on the grating layer $B^{g}:=\left.B\right|_{\Omega_{\delta}}$ and $b^{g}:=\left.b\right|_{\Omega_{\delta}}$. We recall that $B^{g}$ and $b^{g}$ are assumed not to depend on $z$.

In the appendix we perform an asymptotic analysis that allows us to find the following transmission conditions relating $u_{n}^{+}$and $u_{n}^{-}$for $n=0$ and 1 and for all $x \in(0, L)$ :

$$
\left\{\begin{array}{l}
{\left[u_{0}\right](x)=0} \\
{\left[B \frac{\partial u_{0}}{\partial z}\right](x)=0} \\
{\left[u_{1}\right](x)=\left(\frac{1}{B^{g}(x)}-\left\langle\frac{1}{B}\right\rangle\right)\left\langle B \frac{\partial u_{0}}{\partial z}\right\rangle(x),} \\
{\left[B \frac{\partial u_{1}}{\partial z}\right](x)=-\frac{\partial}{\partial x}\left(\left(B^{g}(x)-\langle B\rangle\right) \frac{\partial\left\langle u_{0}\right\rangle}{\partial x}(x)\right)-k_{0}^{2}\left(b^{g}(x)-\langle b\rangle\right)\left\langle u_{0}\right\rangle(x) .}
\end{array}\right.
$$


Adding these transmission conditions to equations (3.2), we are led to the following problems to determine $u_{0}$ and $u_{1}$, respectively:

$$
\begin{aligned}
& \begin{cases}\nabla \cdot\left(B^{ \pm}(x, z) \nabla u_{0}^{ \pm}(x, z)\right)+k_{0}^{2} b^{ \pm}(x, z) u_{0}^{ \pm}(x, z)=0 & \text { in } \Omega^{ \pm}, \\
{\left[u_{0}\right](x)=0} & \text { on } \Gamma, \\
{\left[B \frac{\partial u_{0}}{\partial z}\right](x)=0} & \text { on } \quad \Gamma, \\
u_{0}^{ \pm}(L, z)=e^{i \alpha L} u_{0}^{ \pm}(0, z), & z \in\left(-L_{m}, L_{d}\right), \\
\frac{\partial u_{0}^{ \pm}}{\partial x}(L, z)=e^{i \alpha L} \frac{\partial u_{0}^{ \pm}}{\partial x}(0, z), & z \in\left(-L_{m}, L_{d}\right), \\
B^{-} \frac{\partial u_{0}^{-}}{\partial z}(x, z)=\left(T^{-} u_{0}^{-}\right)(x, z) & \text { on } \Gamma^{-}, \\
B^{+} \frac{\partial u_{0}^{+}}{\partial z}(x, z)-B^{+} \frac{\partial u_{\text {inc }}}{\partial z}(x, z)=\left(T^{+} u_{0}^{+}\right)(x, z)-\left(T^{+} u_{\text {inc }}\right)(x, z) & \text { on } \Gamma^{+} ; \\
\nabla \cdot\left(B^{ \pm}(x, z) \nabla u_{1}^{ \pm}(x, z)\right)+k_{0}^{2} b^{ \pm}(x, z) u_{1}^{ \pm}(x, z)=0 & \text { in } \Omega^{ \pm}, \\
{\left[u_{1}\right](x)=\left(\frac{1}{B^{g}(x)}-\left\langle\frac{1}{B}\right\rangle\right)\left\langle B \frac{\partial u_{0}}{\partial z}\right\rangle(x)} & \text { on } \Gamma, \\
{\left[B \frac{\partial u_{1}}{\partial z}\right](x)=-\frac{\partial}{\partial x}\left(\left(B^{g}(x)-\langle B\rangle\right) \frac{\partial\left\langle u_{0}\right\rangle}{\partial x}(x)\right)-k_{0}^{2}\left(b^{g}(x)-\langle b\rangle\right)\left\langle u_{0}\right\rangle(x)} & \text { on } \quad \Gamma, \\
u_{1}^{ \pm}(L, z)=e^{i \alpha L} u_{1}^{ \pm}(0, z), & z \in\left(-L_{m}, L_{d}\right), \\
\frac{\partial u_{1}^{ \pm}}{\partial x}(L, z)=e^{i \alpha L} \frac{\partial u_{1}^{ \pm}}{\partial x}(0, z), & z \in\left(-L_{m}, L_{d}\right), \\
B^{ \pm} \frac{\partial u_{1}^{ \pm}}{\partial z}(x, z)=\left(T^{ \pm} u_{1}^{ \pm}\right)(x, z) & \text { on } \Gamma^{ \pm} .\end{cases}
\end{aligned}
$$

To complete the derivation, it is also shown in the appendix that $U_{0}$ and $U_{1}$ into the grating are given by

$$
\left.\begin{array}{l}
U_{0}(x, \xi)=u_{0}(x, 0), \\
U_{1}(x, \xi)=\left\langle u_{1}\right\rangle(x)+\left(\frac{\xi}{B^{g}(x)}+\frac{1}{4}\left[\frac{1}{B}\right]\right)\left\langle B \frac{\partial u_{0}}{\partial z}\right\rangle(x),
\end{array}\right\} \quad x \in(0, L), \quad|\xi| \leq \frac{1}{2} .
$$

\section{ERror estimates}

In this section, we estimate the error between the exact solution $u$ and its first-order far field approximation

$$
u_{\delta, 1}(x, z):=u_{0}^{ \pm}(x, z)+\delta u_{1}^{ \pm}(x, z) .
$$

For the forthcoming analysis, we will also use the second-order approximation

$$
u_{\delta, 2}(x, z):=u_{0}^{ \pm}(x, z)+\delta u_{1}^{ \pm}(x, z)+\delta^{2} u_{2}^{ \pm}(x, z)
$$

and the corresponding one for the near field:

$$
U_{\delta, 2}(x, z):=U_{0}^{\delta}(x, z)+\delta U_{1}^{\delta}(x, z)+\delta^{2} U_{2}^{\delta}(x, z) .
$$

We proceed as in $[8]$ and introduce a smooth cut-off function $\chi \in \mathbb{C}^{\infty}(\mathbb{R})$ such that

$$
\chi(\xi)= \begin{cases}1, & \text { if }|\xi| \leq 1 \\ 0, & \text { if }|\xi| \geq 2 .\end{cases}
$$


Then, denoting as above $\chi^{\delta}(z):=\chi\left(\frac{z}{\delta}\right)$, we introduce the global approximate solution

$$
\widetilde{u}_{\delta}(x, z):=\left(1-\chi^{\delta}(z)\right) u_{\delta, 2}(x, z)+\chi^{\delta}(z) U_{\delta, 2}(x, z) \quad(x, z) \in \Omega .
$$

Let us remark that $U_{\delta, 2}(x, z)$ is actually defined only for $|z| \leq 2 \delta$. However, any smooth extension can be used to define properly $\widetilde{u}_{\delta}$. In fact, since in this definition $U_{\delta, 2}$ appears multiplied by $\chi^{\delta}$, which vanishes for $|z| \geq 2 \delta, \widetilde{u}_{\delta}$ does not depend on the particular extension (which we still denote $U_{\delta, 2}$ ). Moreover, according to Assumption 3.2, $\chi^{\delta} U_{\delta, 2} \in H_{\alpha}^{1}(\Omega)$.

In general, $u_{\delta, 2}$ does not lie in $H_{\alpha}^{1}(\Omega)$, but $\left(1-\chi^{\delta}\right) u_{\delta, 2}$ does. In fact, in spite of the fact that $u_{\delta, 2}$ may have a jump on $\Gamma$, this does not affect the smoothness of $\left(1-\chi^{\delta}\right) u_{\delta, 2}$ since $\left(1-\chi^{\delta}\right)$ vanishes for $|z| \leq \delta$. Moreover, according to Assumption 3.1, $\left(1-\chi^{\delta}\right) u_{\delta, 2}$ is infinitely differentiable in the whole of $\Omega$.

Since in what follows we will have to deal with functions like $u_{\delta, 2}$ that are smooth in $\Omega^{+}$and $\Omega^{-}$but not in $\Omega$, from now on we will use the following notation: $\int_{\Omega^{ \pm}} f^{ \pm}(x, z) \mathrm{d} x \mathrm{~d} z=\int_{\Omega^{+}} f^{+}(x, z) \mathrm{d} x \mathrm{~d} z+\int_{\Omega^{-}} f^{+}(x, z) \mathrm{d} x \mathrm{~d} z$ and $\int_{\Gamma^{ \pm}} g^{ \pm}(x) \mathrm{d} x=\int_{\Gamma^{+}} g^{+}(x) \mathrm{d} x+\int_{\Gamma^{-}} g^{+}(x) \mathrm{d} x$.

Now, since from Assumption 2.1 we have the stability estimate

$$
\left\|u-\widetilde{u}_{\delta}\right\|_{H^{1}(\Omega)} \leq C \sup _{\substack{v \in H_{\alpha}^{1}(\Omega) \\ v \neq 0}} \frac{\left|a\left(u-\widetilde{u}_{\delta}, v\right)\right|}{\|v\|_{H^{1}(\Omega)}},
$$

our next goal is to find a bound for the right-hand side.

Lemma 4.1. For all $v \in H_{\alpha}^{1}(\Omega)$,

$$
a\left(u-\widetilde{u}_{\delta}, v\right)=\varepsilon_{\delta}^{m}(v)+\varepsilon_{\delta}^{c}(v),
$$

where

$$
\varepsilon_{\delta}^{m}(v):=\int_{C_{\delta}} B\left(u_{\delta, 2}-U_{\delta, 2}\right) \nabla \chi^{\delta} \cdot \nabla \bar{v} \mathrm{~d} x \mathrm{~d} z-\int_{C_{\delta}} B \nabla\left(u_{\delta, 2}-U_{\delta, 2}\right) \cdot \nabla \chi^{\delta} \bar{v} \mathrm{~d} x \mathrm{~d} z
$$

and

$$
\varepsilon_{\delta}^{c}(v):=-a\left(U_{\delta, 2}, \chi^{\delta} v\right) .
$$

Terms $\varepsilon_{\delta}^{m}(v)$ and $\varepsilon_{\delta}^{c}(v)$ are called the matching error and the consistency error, respectively.

Proof. Taking into account the definition of $\widetilde{u}_{\delta}$ and (2.7), for all $v \in H_{\alpha}^{1}(\Omega)$ we have that

$$
a\left(u-\widetilde{u}_{\delta}, v\right)=L(v)-a\left(\left(1-\chi^{\delta}\right) u_{\delta, 2}, v\right)-a\left(\chi^{\delta} U_{\delta, 2}, v\right) .
$$

For the second term on the right-hand side, the definition of $a(\cdot, \cdot)$ and straightforward computations lead to

$$
\begin{aligned}
a((1- & \left.\left.\chi^{\delta}\right) u_{\delta, 2}, v\right) \\
= & \int_{\Omega^{ \pm}}\left\{B^{ \pm} \nabla\left(\left(1-\chi^{\delta}\right) u_{\delta, 2}\right) \cdot \nabla \bar{v}-k_{0}^{2} b^{ \pm}\left(1-\chi^{\delta}\right) u_{\delta, 2} \bar{v}\right\} \mathrm{d} x \mathrm{~d} z-\int_{\Gamma^{ \pm}}\left(T^{ \pm}\left(\left(1-\chi^{\delta}\right) u_{\delta, 2}\right)\right) \bar{v} \mathrm{~d} x \\
= & \int_{\Omega^{ \pm}} B^{ \pm} u_{\delta, 2} \nabla\left(1-\chi^{\delta}\right) \cdot \nabla \bar{v} \mathrm{~d} x \mathrm{~d} z-\int_{\Omega^{ \pm}} B^{ \pm} \nabla u_{\delta, 2} \cdot \nabla\left(1-\chi^{\delta}\right) \bar{v} \mathrm{~d} x \mathrm{~d} z \\
& \quad+\int_{\Omega^{ \pm}}\left\{B^{ \pm} \nabla u_{\delta, 2} \cdot \nabla\left(\left(1-\chi^{\delta}\right) \bar{v}\right)-k_{0}^{2} b^{ \pm} u_{\delta, 2}\left(1-\chi^{\delta}\right) \bar{v}\right\} \mathrm{d} x \mathrm{~d} z-\int_{\Gamma^{ \pm}}\left(T^{ \pm} u_{\delta, 2}\right)\left(1-\chi^{\delta}\right) \bar{v} \mathrm{~d} x .
\end{aligned}
$$

Now, multiplying the first equation in $(3.2)$ by $\left(1-\chi^{\delta}\right)$, integrating by parts separately in $\Omega^{+}$and $\Omega^{-}$and using the boundary conditions from (3.2), we obtain

$$
\begin{aligned}
\int_{\Omega^{ \pm}} & \left\{B^{ \pm} \nabla u_{\delta, 2} \cdot \nabla\left(\left(1-\chi^{\delta}\right) \bar{v}\right)-k_{0}^{2} b^{ \pm} u_{\delta, 2}\left(1-\chi^{\delta}\right) \bar{v}\right\} \mathrm{d} x \mathrm{~d} z-\int_{\Gamma^{ \pm}}\left(T^{ \pm} u_{\delta, 2}\right)\left(1-\chi^{\delta}\right) \bar{v} \mathrm{~d} x \\
= & \int_{\Gamma^{+}}\left(B^{+} \frac{\partial u_{\text {inc }}}{\partial z}-T^{+} u_{\text {inc }}\right)\left(1-\chi^{\delta}\right) \bar{v} \mathrm{~d} x=L(v) .
\end{aligned}
$$


Let us emphasize that in spite of the fact that $B^{ \pm} \nabla u_{\delta, 2}$ is in general discontinuous on $\Gamma$, no jump across this curve appears from the integration by parts because $\left(1-\chi^{\delta}\right)$ vanishes for $|z| \leq \delta$ and thus on $\Gamma$.

Then, substituting the above equation into (4.9) we have that

$$
a\left(\left(1-\chi^{\delta}\right) u_{\delta, 2}, v\right)=\int_{\Omega^{ \pm}} B^{ \pm} u_{\delta, 2} \nabla\left(1-\chi^{\delta}\right) \cdot \nabla \bar{v} \mathrm{~d} x \mathrm{~d} z-\int_{\Omega^{ \pm}} B^{ \pm} \nabla u_{\delta, 2} \cdot \nabla\left(1-\chi^{\delta}\right) \bar{v} \mathrm{~d} x \mathrm{~d} z+L(v) .
$$

Next, computations similar to those that lead to (4.9) yield

$$
a\left(\chi^{\delta} U_{\delta, 2}, v\right)=a\left(U_{\delta, 2}, \chi^{\delta} v\right)+\int_{\Omega^{ \pm}} B^{ \pm} U_{\delta, 2} \nabla \chi^{\delta} \cdot \nabla \bar{v} \mathrm{~d} x \mathrm{~d} z-\int_{\Omega^{ \pm}} B^{ \pm} \nabla U_{\delta, 2} \cdot \nabla \chi^{\delta} \bar{v} \mathrm{~d} x \mathrm{~d} z
$$

Finally, substituting the last two equations into (4.8) we obtain

$$
a\left(u-\widetilde{u}_{\delta}, v\right)=\int_{\Omega^{ \pm}} B^{ \pm}\left(u_{\delta, 2}-U_{\delta, 2}\right) \nabla \chi^{\delta} \cdot \nabla \bar{v} \mathrm{~d} x \mathrm{~d} z-\int_{\Omega^{ \pm}} B^{ \pm} \nabla\left(u_{\delta, 2}-U_{\delta, 2}\right) \cdot \nabla \chi^{\delta} \bar{v} \mathrm{~d} x \mathrm{~d} z-a\left(U_{\delta, 2}, \chi^{\delta} v\right) .
$$

Since $\nabla \chi^{\delta}$ vanishes out of $C_{\delta}$, the above equation and definitions (4.6) and (4.7) allow us to end the proof.

The following estimates are similar to those used in [8]. For the sake of completeness, we include the corresponding proofs.

Lemma 4.2. Let $O_{\delta}:=\{(x, z) \in \Omega:|z| \leq 2 \delta\}$. Then, for all $v \in H_{\alpha}^{1}(\Omega)$,

$$
\begin{aligned}
\|v\|_{L^{2}\left(O_{\delta}\right)} & \leq C \sqrt{\delta}\|v\|_{H^{1}(\Omega)}, \\
\|v\|_{L^{1}\left(O_{\delta}\right)} & \leq C \delta\|v\|_{H^{1}(\Omega)}, \\
\|\nabla v\|_{L^{1}\left(O_{\delta}\right)} & \leq C \sqrt{\delta}\|v\|_{H^{1}(\Omega)} .
\end{aligned}
$$

Proof. We use a density argument. For $v$ smooth enough we write

$$
v(x, z)=v(x, 0)+\int_{0}^{z} \frac{\partial v}{\partial z}(x, t) \mathrm{d} t \quad \forall(x, z) \in O_{\delta} .
$$

Then,

$$
\int_{O_{\delta}}|v(x, z)|^{2} \mathrm{~d} x \mathrm{~d} z \leq 2 \int_{O_{\delta}}|v(x, 0)|^{2} \mathrm{~d} x \mathrm{~d} z+\left.2 \int_{O_{\delta}}\left|\int_{0}^{z}\right| \frac{\partial v}{\partial z}(x, t)\right|^{2} \mathrm{~d} t \mid \mathrm{d} x \mathrm{~d} z .
$$

For the first term we have

$$
\int_{O_{\delta}}|v(x, 0)|^{2} \mathrm{~d} x \mathrm{~d} z=4 \delta \int_{0}^{L}|v(x, 0)|^{2} \mathrm{~d} x=4 \delta\|v\|_{L^{2}(\Gamma)}^{2} \leq C \delta\|v\|_{H^{1}(\Omega)}^{2}
$$

and for the second one

$$
\left.\int_{O_{\delta}}\left|\int_{0}^{z}\right| \frac{\partial v}{\partial z}(x, t)\right|^{2} \mathrm{~d} t \mid \mathrm{d} x \mathrm{~d} z \leq 4 \delta \int_{0}^{L}\left(\int_{-2 \delta}^{2 \delta}\left|\frac{\partial v}{\partial z}(x, t)\right|^{2} \mathrm{~d} t\right) \mathrm{d} x \leq C \delta\|v\|_{H^{1}(\Omega)}^{2} .
$$

Thus, the first estimate of the lemma follows from the last three inequalities.

The second estimate follows from Cauchy-Schwarz inequality and the previous one. The last one follows from Cauchy-Schwarz inequality.

For the matching error we have the following estimate. 
Lemma 4.3. For all $v \in H_{\alpha}^{1}(\Omega)$,

$$
\varepsilon_{\delta}^{m}(v) \leq C \delta^{2}\|v\|_{H^{1}(\Omega)} .
$$

Proof. Let $v \in H_{\alpha}^{1}(\Omega)$. The far field terms $u_{n}$ and the near field terms $U_{n}^{\delta}$ have been assumed to lie in $\mathcal{C}^{\infty}\left(C_{\delta}\right)$ (cf. Assumptions 3.1 and 3.2). Then, from the definition (4.6) of $\varepsilon_{\delta}^{m}(v)$ and Hölder inequality, since $\chi^{\delta}$ does not depend on $x$ and the support of its gradient is contained in $C_{\delta}$, we have that

$$
\left|\varepsilon_{\delta}^{m}(v)\right| \leq\|B\|_{L^{\infty}(\Omega)}\left\|\nabla \chi^{\delta}\right\|_{L^{\infty}(\mathbb{R})}\left\{\left\|\frac{\partial v}{\partial z}\right\|_{L^{1}\left(C_{\delta}\right)}\left\|u_{\delta, 2}-U_{\delta, 2}\right\|_{L^{\infty}\left(C_{\delta}\right)}+\left\|\frac{\partial}{\partial z}\left(u_{\delta, 2}-U_{\delta, 2}\right)\right\|_{L^{\infty}\left(C_{\delta}\right)}\|v\|_{L^{1}\left(C_{\delta}\right)}\right\} .
$$

In what follows, we estimate each of the terms on the right-hand side above.

- For the cut-off function, since $\nabla \chi^{\delta}=\frac{1}{\delta} \nabla \chi$, we have

$$
\left\|\nabla \chi^{\delta}\right\|_{L^{\infty}(\mathbb{R})}=\frac{1}{\delta}\|\nabla \chi\|_{L^{\infty}(\mathbb{R})} \leq \frac{C}{\delta} .
$$

- To estimate $\left\|u_{\delta, 2}-U_{\delta, 2}\right\|_{L^{\infty}\left(C_{\delta}\right)}$, for the far field approximation $u_{\delta, 2}$, we use Taylor's formula with integral remainder for each $u_{n}^{ \pm}, n=0,1,2$. Then, from (4.2) we have

$$
u_{\delta, 2}(x, z)=\sum_{n=0}^{2} \delta^{n}\left\{\sum_{i=0}^{2-n} \frac{z^{i}}{i !} \frac{\partial^{i} u_{n}^{ \pm}}{\partial z^{i}}(x, 0)+\int_{0}^{z} \frac{\partial^{3-n} u_{n}^{ \pm}}{\partial z^{3-n}}(x, t) \frac{(z-t)^{2-n}}{(2-n) !} \mathrm{d} t\right\} .
$$

For the near field, from (3.7) and the matching conditions (3.8), in the overlapping zones we have

$$
U_{n}^{\delta}(x, z)=\sum_{i=0}^{n} \frac{z^{i}}{\delta^{i} i !} \frac{\partial^{i} u_{n-i}^{ \pm}}{\partial z^{i}}(x, 0) .
$$

Hence, from (4.3),

$$
\begin{aligned}
U_{\delta, 2}(x, z) & =\sum_{n=0}^{2} \delta^{n}\left(\sum_{i=0}^{n} \frac{z^{i}}{\delta^{i} i !} \frac{\partial^{i} u_{n-i}^{ \pm}}{\partial z^{i}}(x, 0)\right)=\sum_{n=0}^{2} \sum_{i=0}^{n} \delta^{n-i} \frac{z^{i}}{i !} \frac{\partial^{i} u_{n-i}^{ \pm}}{\partial z^{i}}(x, 0) \\
& =\sum_{i=0}^{2} \sum_{n=i}^{2} \delta^{n-i} \frac{z^{i}}{i !} \frac{\partial^{i} u_{n-i}^{ \pm}}{\partial z^{i}}(x, 0)=\sum_{i=0}^{2} \sum_{j=0}^{2-i} \delta^{j} \frac{z^{i}}{i !} \frac{\partial^{i} u_{j}^{ \pm}}{\partial z^{i}}(x, 0)=\sum_{j=0}^{2} \sum_{i=0}^{2-j} \delta^{j} \frac{z^{i}}{i !} \frac{\partial^{i} u_{j}^{ \pm}}{\partial z^{i}}(x, 0) .
\end{aligned}
$$

Then, subtracting (4.13) from (4.12), we obtain

$$
u_{\delta, 2}(x, z)-U_{\delta, 2}(x, z)=\sum_{n=0}^{2} \delta^{n} \int_{0}^{z} \frac{\partial^{3-n} u_{n}^{ \pm}}{\partial z^{3-n}}(x, t) \frac{(z-t)^{2-n}}{(2-n) !} \mathrm{d} t .
$$

Now, since the far field terms and their derivatives are bounded in $C_{\delta}$ (cf. Assumption 3.1), we estimate the integral above as follows:

$$
\left|\int_{0}^{z} \frac{\partial^{3-n} u_{n}^{ \pm}}{\partial z^{3-n}}(x, t) \frac{(z-t)^{2-n}}{(2-n) !} \mathrm{d} t\right| \leq C \delta^{3-n} \quad \forall(x, z) \in C_{\delta} .
$$

Then,

$$
\left\|u_{\delta, 2}-U_{\delta, 2}\right\|_{L^{\infty}\left(C_{\delta}\right)} \leq C \delta^{3}
$$


- For $\left\|\frac{\partial}{\partial z}\left(u_{\delta, 2}-U_{\delta, 2}\right)\right\|_{L^{\infty}\left(C_{\delta}\right)}$, differentiating (4.14) with respect to $z$ we have

$$
\frac{\partial u_{\delta, 2}}{\partial z}(x, z)-\frac{\partial U_{\delta, 2}}{\partial z}(x, z)=\sum_{n=0}^{1} \delta^{n} \int_{0}^{z} \frac{\partial^{3-n} u_{n}^{ \pm}}{\partial z^{3-n}}(x, t) \frac{(z-t)^{1-n}}{(1-n) !} \mathrm{d} t .
$$

Since the far field terms and their derivatives are bounded in $C_{\delta}$, we estimate the integral as follows:

$$
\left|\int_{0}^{z} \frac{\partial^{3-n} u_{n}^{ \pm}(x, t)}{\partial z^{3-n}} \frac{(z-t)^{1-n}}{(1-n) !} \mathrm{d} t\right| \leq C \delta^{2-n} \quad \forall(x, z) \in C_{\delta} .
$$

Therefore,

$$
\left\|\frac{\partial\left(u_{\delta, 2}-U_{\delta, 2}\right)}{\partial z}\right\|_{L^{\infty}\left(C_{\delta}\right)} \leq C \delta^{2} .
$$

Finally, using (4.11), (4.15), (4.16) and Lemma 4.2 to estimate all terms in (4.10), we conclude the proof.

For the consistency error (4.7), we have the following estimate.

Lemma 4.4. For all $v \in H_{\alpha}^{1}(\Omega)$,

$$
\left|\varepsilon_{\delta}^{c}(v)\right| \leq C \delta^{2}\|v\|_{H^{1}(\Omega)} .
$$

Proof. Integrating by parts and using the quasi-periodic character of $U_{\delta, 2}$ and $v$ and the fact that the support of $\chi^{\delta}$ is contained in $O_{\delta}$, we have that

$$
\varepsilon_{\delta}^{c}(v)=-\int_{\Omega}\left(B \nabla U_{\delta, 2} \cdot \nabla\left(\chi^{\delta} \bar{v}\right)-k_{0}^{2} b U_{\delta, 2} \chi^{\delta} \bar{v}\right) \mathrm{d} x \mathrm{~d} z=\int_{O_{\delta}}\left(\nabla \cdot\left(B \nabla U_{\delta, 2}\right)+k_{0}^{2} b U_{\delta, 2}\right) \chi^{\delta} \bar{v} \mathrm{~d} x \mathrm{~d} z .
$$

Now, recalling the definition (4.3) and using (3.4) with $\xi=\frac{z}{\delta}$ and the first equation from (3.5), we obtain

$$
\begin{aligned}
\nabla \cdot & \left(B(x, z) \nabla U_{\delta, 2}(x, z)\right)+k_{0}^{2} b(x, z) U_{\delta, 2}(x, z) \\
= & \frac{1}{\delta^{2}} \frac{\partial}{\partial \xi}\left(B(x, \delta \xi) \frac{\partial U_{0}}{\partial \xi}(x, \xi)\right)+\frac{1}{\delta} \frac{\partial}{\partial \xi}\left(B(x, \delta \xi) \frac{\partial U_{1}}{\partial \xi}(x, \xi)\right)+\frac{\partial}{\partial \xi}\left(B(x, \delta \xi) \frac{\partial U_{2}}{\partial \xi}(x, \xi)\right) \\
& +\frac{\partial}{\partial x}\left(B(x, \delta \xi) \frac{\partial U_{0}}{\partial x}(x, \xi)\right)+k_{0}^{2} b(x, \delta \xi) U_{0}(x, \xi)+\delta\left(\frac{\partial}{\partial x}\left(B(x, \delta \xi) \frac{\partial U_{1}}{\partial x}(x, \xi)\right)+k_{0}^{2} b(x, \delta \xi) U_{1}(x, \xi)\right) \\
& +\delta^{2}\left(\frac{\partial}{\partial x}\left(B(x, \delta \xi) \frac{\partial U_{2}}{\partial x}(x, \xi)\right)+k_{0}^{2} b(x, \delta \xi) U_{2}(x, \xi)\right) \\
= & \delta\left(\frac{\partial}{\partial x}\left(B(x, z) \frac{\partial U_{1}^{\delta}}{\partial x}(x, z)\right)+k_{0}^{2} b(x, z) U_{1}^{\delta}(x, z)\right)+\delta^{2}\left(\frac{\partial}{\partial x}\left(B(x, z) \frac{\partial U_{2}^{\delta}}{\partial x}(x, z)\right)+k_{0}^{2} b(x, z) U_{2}^{\delta}(x, z)\right)
\end{aligned}
$$

Then,

$$
\varepsilon_{\delta}^{c}(v)=\delta \varepsilon_{\delta}^{c, 1}(v)+\delta^{2} \varepsilon_{\delta}^{c, 2}(v)
$$

where

$$
\varepsilon_{\delta}^{c, i}(v):=\int_{O_{\delta}}\left(\frac{\partial}{\partial x}\left(B \frac{\partial U_{i}^{\delta}}{\partial x}\right)+k_{0}^{2} b U_{i}^{\delta}\right) \chi^{\delta} \bar{v} \mathrm{~d} x \mathrm{~d} z, \quad i=1,2 .
$$

From (2.3) (for the $s$-polarization) or (2.4) (for the $p$-polarization) combined with the additional assumption that $\varepsilon_{r}^{g}(x)$ is infinitely differentiable in $[0, L[$, we have that the $x$-derivative of $B$ is bounded in $\Omega$. So are the $x$-derivatives of $U_{i}^{\delta}$ too (cf. Lem. A.1 in the appendix). Then,

$$
\left|\varepsilon_{\delta}^{c, i}(v)\right| \leq C\|v\|_{L^{1}\left(O_{\delta}\right)} \leq C \delta\|v\|_{H^{1}(\Omega)}, \quad i=1,2,
$$

where we have used Lemma 4.2 for the last inequality. Hence, the lemma follows from (4.17). 
Now, we are in a position to write the main approximation result of the paper.

Theorem 4.5. Let $u$ be the solution of problem (2.7) and $u_{\delta, 1}$ its far field approximation (4.1). Then, given $\gamma>0$, there exist a constant $C>0$ such that for all $\delta<\frac{\gamma}{2}$,

$$
\left\|u-u_{\delta, 1}\right\|_{H^{1}\left(\widehat{\Omega}_{\gamma}\right)} \leq C \delta^{2}
$$

where $\widehat{\Omega}_{\gamma}:=\{(x, z) \in \Omega:|z|>\gamma\}$.

Proof. From (4.4) and Lemmas 4.1, 4.3 and 4.4, we write

$$
\left\|u-\widetilde{u}_{\delta}\right\|_{H^{1}(\Omega)} \leq C \sup _{\substack{v \in H_{\alpha}^{1}(\Omega) \\ v \neq 0}} \frac{\left|a\left(u-\widetilde{u}_{\delta}, v\right)\right|}{\|v\|_{H^{1}(\Omega)}} \leq C \sup _{\substack{v \in H_{\alpha}^{1}(\Omega) \\ v \neq 0}} \frac{\left|\varepsilon_{\delta}^{m}(v)\right|+\left|\varepsilon_{\delta}^{c}(v)\right|}{\|v\|_{H^{1}(\Omega)}} \leq C \delta^{2} .
$$

Now, given $\gamma>0$, for all $\delta<\frac{\gamma}{2}, \widetilde{u}_{\delta}=u_{\delta, 2}$ in $\widehat{\Omega}_{\gamma}$. Then, from the definition (4.2) of $u_{\delta, 2}$ and using that $u_{n}^{ \pm} \in H_{\alpha}^{1}\left(\Omega_{\delta}^{ \pm}\right)(c f$. Assumption 3.1), we have that

$$
\left\|\widetilde{u}_{\delta}-u_{\delta, 1}\right\|_{H^{1}\left(\widehat{\Omega}_{\gamma}\right)} \leq \delta^{2}\left\|u_{2}^{+}\right\|_{H^{1}\left(\Omega^{+}\right)}+\delta^{2}\left\|u_{2}^{-}\right\|_{H^{1}\left(\Omega^{-}\right)} \leq C \delta^{2}
$$

and, hence,

$$
\left\|u-u_{\delta, 1}\right\|_{H^{1}\left(\widehat{\Omega}_{\gamma}\right)} \leq\left\|u-\widetilde{u}_{\delta}\right\|_{H^{1}\left(\widehat{\Omega}_{\gamma}\right)}+\left\|\widetilde{u}_{\delta}-u_{\delta, 1}\right\|_{H^{1}\left(\widehat{\Omega}_{\gamma}\right)} \leq C \delta^{2}
$$

\section{IMPLEMENTATION}

In order to avoid solving separately problems (3.10) and (3.11), it is possible to approximate directly $u_{\delta, 1}=$ $u_{0}+\delta u_{1}$ up to $\delta^{2}$-terms. With this purpose, we use equations (3.9) to write

$$
\left[u_{\delta, 1}\right]=\left[u_{0}\right]+\delta\left[u_{1}\right]=\delta\left(\frac{1}{B^{g}(x)}-\left\langle\frac{1}{B}\right\rangle\right)\left\langle B \frac{\partial u_{\delta, 1}}{\partial z}\right\rangle-\delta^{2}\left(\frac{1}{B^{g}(x)}-\left\langle\frac{1}{B}\right\rangle\right)\left\langle B \frac{\partial u_{1}}{\partial z}\right\rangle
$$

and

$$
\begin{aligned}
{\left[B \frac{\partial u_{\delta, 1}}{\partial z}\right]=\left[B \frac{\partial u_{0}}{\partial z}\right]+\delta\left[B \frac{\partial u_{1}}{\partial z}\right]=} & -\delta\left\{\frac{\partial}{\partial x}\left(\left(B^{g}(x)-\langle B\rangle\right) \frac{\partial}{\partial x}\right)+k_{0}^{2}\left(b^{g}(x)-\langle b\rangle\right)\right\}\left\langle u_{\delta, 1}\right\rangle \\
& +\delta^{2}\left\{\frac{\partial}{\partial x}\left(\left(\left(B^{g}(x)-\langle B\rangle\right) \frac{\partial}{\partial x}\right)+k_{0}^{2}\left(b^{g}(x)-\langle b\rangle\right)\right\}\left\langle u_{1}\right\rangle .\right.
\end{aligned}
$$

Since $u_{0}^{ \pm}$and $u_{1}^{ \pm}$satisfy (3.2), so does $u_{0}^{ \pm}+\delta u_{1}^{ \pm}$. To obtain a well posed problem we must complement these equations with appropriate jump conditions on $\Gamma$. With this end, we use the two above equations, neglecting 
the $\delta^{2}$ terms. Therefore, we are led to the following alternative problem, whose solution we denote by $\widehat{u}_{\delta, 1}^{ \pm}$:

$$
\begin{cases}\nabla \cdot\left(B^{ \pm} \nabla \widehat{u}_{\delta, 1}^{ \pm}(x, z)\right)+k_{0}^{2} b^{ \pm} \widehat{u}_{\delta, 1}^{ \pm}(x, z)=0 & \text { in } \Omega^{ \pm}, \\ {\left[\widehat{u}_{\delta, 1}\right](x)=\delta\left(\frac{1}{B^{g}(x)}-\left\langle\frac{1}{B}\right\rangle\right)\left\langle B \frac{\partial \widehat{u}_{\delta, 1}}{\partial z}\right\rangle(x)} & \text { on } \Gamma, \\ {\left[B \frac{\partial u_{1}}{\partial z}\right](x)=-\delta \frac{\partial}{\partial x}\left(\left(B^{g}(x)-\langle B\rangle\right) \frac{\partial\left\langle\widehat{u}_{\delta, 1}\right\rangle}{\partial x}(x)\right)-\delta k_{0}^{2}\left(b^{g}(x)-\langle b\rangle\right)\left\langle\widehat{u}_{\delta, 1}\right\rangle(x)} & \text { on } \Gamma, \\ \widehat{u}_{\delta, 1}^{ \pm}(L, z)=e^{i \alpha L} \widehat{u}_{\delta, 1}^{ \pm}(0, z) & z \in\left(-L_{m}, L_{d}\right), \\ \frac{\partial \widehat{u}_{\delta, 1}^{ \pm}}{\partial x}(L, z)=e^{i \alpha L} \frac{\partial \widehat{u}_{\delta, 1}^{ \pm}}{\partial x}(L, z) & z \in\left(-L_{m}, L_{d}\right), \\ B^{-} \frac{\partial \widehat{u}_{\delta, 1}^{-}}{\partial z}(x, z)=T^{-} \widehat{u}_{\delta, 1}^{-}(x, z) & \text { on } \Gamma^{-}, \\ B^{+} \frac{\partial \widehat{u}_{\delta, 1}^{+}}{\partial z}(x, z)-B^{+} \frac{\partial u_{\text {inc }}}{\partial z}(x, z)=T^{+}\left(\widehat{u}_{\delta, 1}^{+}(x, z)-u_{\text {inc }}(x, z)\right) & \text { on } \Gamma^{+} .\end{cases}
$$

\subsection{Variational formulation}

To implement a FEM for this asymptotic model, we need a variational formulation of problem (5.1). With this end in mind, consider the Hilbert space

$$
V:=\left\{v \in L^{2}(\Omega): v^{-} \in H_{\alpha}^{1}\left(\Omega^{-}\right), v^{+} \in H_{\alpha}^{1}\left(\Omega^{+}\right) \quad \text { and } \quad\langle v\rangle \in H_{\alpha}^{1}(\Gamma)\right\}
$$

endowed with the norm defined by

$$
\|v\|_{V}^{2}:=\|v\|_{H^{1}\left(\Omega^{-}\right)}^{2}+\|v\|_{H^{1}\left(\Omega^{+}\right)}^{2}+\|\langle v\rangle\|_{H^{1}(\Gamma)}^{2}
$$

Multiplying the first equation in (5.1) by $\bar{v} \in V$ and integrating by parts, we obtain

$$
\int_{\Omega^{ \pm}}\left(B^{ \pm} \nabla \widehat{u}_{\delta, 1}^{ \pm} \cdot \nabla \bar{v}^{ \pm}-k_{0}^{2} b^{ \pm} \widehat{u}_{\delta, 1}^{ \pm} \bar{v}^{ \pm}\right) \mathrm{d} x \mathrm{~d} z+\int_{\partial \Omega^{+}} B^{+} \frac{\partial \widehat{u}_{\delta, 1}^{+}}{\partial n} \bar{v}^{+} \mathrm{d} s+\int_{\partial \Omega^{-}} B^{-} \frac{\partial \widehat{u}_{\delta, 1}^{-}}{\partial n} \bar{v}^{-} \mathrm{d} s=0
$$

Because of the quasi-periodic character of $v$, the integrals on $\partial \Omega^{ \pm}$reduce to $\Gamma$ and $\Gamma^{ \pm}$. For the former we have

$$
\int_{\Gamma} B^{+} \frac{\partial \widehat{u}_{\delta, 1}^{+}}{\partial z} \bar{v}^{+} \mathrm{d} x-\int_{\Gamma} B^{-} \frac{\partial \widehat{u}_{\delta, 1}^{-}}{\partial z} \bar{v}^{-} \mathrm{d} x=\int_{\Gamma}\left[B \frac{\partial \widehat{u}_{\delta, 1}}{\partial z} \bar{v}\right] \mathrm{d} x=\int_{\Gamma}\left[B \frac{\partial \widehat{u}_{\delta, 1}}{\partial z}\right]\langle\bar{v}\rangle \mathrm{d} x+\int_{\Gamma}\left\langle B \frac{\partial \widehat{u}_{\delta, 1}}{\partial z}\right\rangle[\bar{v}] \mathrm{d} x
$$

For the second term on the right-hand side above, using the second equation from (5.1) we have that

$$
\left\langle B \frac{\partial \widehat{u}_{\delta, 1}}{\partial z}\right\rangle(x)=\frac{1}{\delta}\left(\frac{1}{B^{g}(x)}-\left\langle\frac{1}{B}\right\rangle\right)^{-1}\left[\widehat{u}_{\delta, 1}\right](x)
$$


provided $\frac{1}{B^{g}(x)} \neq\left\langle\frac{1}{B}\right\rangle$. Then, using the third equation from (5.1) and integrating by parts yield

$$
\begin{aligned}
& \int_{\Gamma} B^{+} \frac{\partial \widehat{u}_{\delta, 1}^{+}}{\partial z} \bar{v}^{+} \mathrm{d} x-\int_{\Gamma} B^{-} \frac{\partial \widehat{u}_{\delta, 1}^{-}}{\partial z} \bar{v}^{-} \mathrm{d} x \\
&=-\delta \int_{\Gamma} \frac{\partial}{\partial x}\left(\left(B^{g}(x)-\langle B\rangle\right) \frac{\partial\left\langle\widehat{u}_{\delta, 1}\right\rangle}{\partial x}\right)\langle\bar{v}\rangle \mathrm{d} x-\delta k_{0}^{2} \int_{\Gamma}\left(b^{g}(x)-\langle b\rangle\right)\left\langle\widehat{u}_{\delta, 1}\right\rangle\langle\bar{v}\rangle \mathrm{d} x \\
&+ \frac{1}{\delta} \int_{\Gamma}\left(\frac{1}{B^{g}(x)}-\left\langle\frac{1}{B}\right\rangle\right)^{-1}\left[\widehat{u}_{\delta, 1}\right][\bar{v}] \mathrm{d} x \\
&= \delta \int_{\Gamma}\left(B^{g}(x)-\langle B\rangle\right) \frac{\partial\left\langle\widehat{u}_{\delta, 1}\right\rangle}{\partial x} \frac{\partial\langle\bar{v}\rangle}{\partial x} \mathrm{~d} x-\delta k_{0}^{2} \int_{\Gamma}\left(b^{g}(x)-\langle b\rangle\right)\left\langle\widehat{u}_{\delta, 1}\right\rangle\langle\bar{v}\rangle \mathrm{d} x \\
&+\frac{1}{\delta} \int_{\Gamma}\left(\frac{1}{B^{g}(x)}-\left\langle\frac{1}{B}\right\rangle\right)^{-1}\left[\widehat{u}_{\delta, 1}\right][\bar{v}] \mathrm{d} x
\end{aligned}
$$

For the integrals on $\Gamma^{+}$and $\Gamma^{-}$, we proceed as we did to derive (2.7) and we arrive at similar terms. Therefore, all together, we are led to the following weak form of problem (5.1): Find $\widehat{u}_{\delta, 1} \in V$ such that

$$
\widehat{a}\left(\widehat{u}_{\delta, 1}, v\right)=L(v) \quad \forall v \in V
$$

where the sesquilinear form $\widehat{a}(\cdot, \cdot)$ is defined for all $w, v \in V$ by

$$
\begin{aligned}
\widehat{a}(w, v):= & \int_{\Omega^{ \pm}}\left(B^{ \pm} \nabla w^{ \pm} \cdot \nabla \bar{v}^{ \pm}-k_{0}^{2} b^{ \pm} w^{ \pm} \bar{v}^{ \pm}\right) \mathrm{d} x \mathrm{~d} z+\delta \int_{\Gamma}\left(B^{g}(x)-\langle B\rangle\right) \frac{\partial\langle w\rangle}{\partial x} \frac{\partial\langle\bar{v}\rangle}{\partial x} \mathrm{~d} x \\
& -\delta k_{0}^{2} \int_{\Gamma}\left(b^{g}(x)-\langle b\rangle\right)\langle w\rangle\langle\bar{v}\rangle \mathrm{d} x+\frac{1}{\delta} \int_{\Gamma}\left(\frac{1}{B^{g}(x)}-\left\langle\frac{1}{B}\right\rangle\right)^{-1}[w][\bar{v}] \mathrm{d} x-\int_{\Gamma^{ \pm}}\left(T^{ \pm} w^{ \pm}\right) \bar{v}^{ \pm} \mathrm{d} x
\end{aligned}
$$

and the linear functional $L$ is the same as in (2.8)

Let us remark that equation (5.2) only makes sense provided $\frac{1}{B^{g}(x)} \neq\left\langle\frac{1}{B}\right\rangle$. In case that this term vanishes identically, the second equation from (5.1) implies that $\left[\widehat{u}_{\delta, 1}\right] \equiv 0$ on $\Gamma$ and this condition should be imposed on the trial and test functions. Therefore, in such a case, the space $V$ must be replaced by

$$
\widetilde{V}:=\left\{v \in H_{\alpha}^{1}(\Omega):\left.v\right|_{\Gamma} \in H_{\alpha}^{1}(\Gamma)\right\}
$$

In particular, this happens for the $s$-polarization, when $B^{g}(x)=B^{+}=B^{-}=1$. In such a case, substituting $B$ and $b$ in terms of the physical parameters, we are led to the following problem: Find $\widehat{u}_{\delta, 1} \in \widetilde{V}$ such that

$$
\widehat{a}_{s}\left(\widehat{u}_{\delta, 1}, v\right)=L(v) \quad \forall v \in \widetilde{V}
$$

where

$$
\widehat{a}_{s}(w, v):=\int_{\Omega^{ \pm}}\left(\nabla w^{ \pm} \cdot \nabla \bar{v}^{ \pm}-k_{0}^{2} \varepsilon_{r}^{ \pm} w^{ \pm} \bar{v}^{ \pm}\right) \mathrm{d} x \mathrm{~d} z-\delta k_{0}^{2} \int_{\Gamma}\left(\varepsilon_{r}^{g}-\left\langle\varepsilon_{r}\right\rangle\right) w \bar{v} \mathrm{~d} x-\int_{\Gamma^{ \pm}}\left(T^{ \pm} w^{ \pm}\right) \bar{v}^{ \pm} \mathrm{d} x, \quad w, v \in \widetilde{V} .
$$

We finish this section by mentioning that, in general, it is not possible to ensure that the variational formulations (5.3) and (5.4) are wellposed. In some situations, the properties of the electric permittivity leads to an elliptic bilinear form, whereas in other cases, the bilinear form is indefinite and wellposedeness holds only up to a sequence of countable frequencies. 


\section{Numerical EXAMPLES}

In this section, we report the results of two numerical tests, to demonstrate numerically the convergence properties of the asymptotic model. We have solved problem (5.3) using standard Lagrange FEM with thirddegree polynomials. For the Dirichlet-to-Neumann operators $T^{+}$and $T^{-}$, we have used a truncated Fourier expansion approach similar to that in [23].

In the examples that follow, we focus on the convergence of the asymptotic model at a fixed wavelength $\lambda_{0}=450 \mathrm{~nm}$. For all examples, we have fixed $L=400 \mathrm{~nm}, L_{d}=131.25 \mathrm{~nm}$, and $L_{m}=56.25 \mathrm{~nm}$. We have chosen values of $\delta$ between $12.5 \mathrm{~nm}$ and $6.103125 \mathrm{e}-03 \mathrm{~nm}$, namely, between $6.67 \%$ and $0.003255 \%$ of the total height $L_{m}+L_{d}=187.5 \mathrm{~nm}$.

We have fixed the angle of incidence to $\theta=0$, since most solar cells are illuminated normally to maximize photonic absorption. The physical parameters have been taken as in [23], where further details can be found: $\varepsilon_{r}^{+}=3.6876$ and $\varepsilon_{r}^{-}=-5.8828+0.6650 \mathrm{i}$.

The domain $\Omega^{+} \cup \Omega^{-}$has been discretized with a triangular mesh with $N_{e}$ triangles and mesh size $h$. For each polarization state, $q=s$ or $q=p$, let $\widehat{u}_{\delta, 1}^{q, h}$ denote the approximate values of the solution to problem (2.7), delivered by the FEM solution of the asymptotic model (5.3) for the $p$-polarization or (5.4) for the $s$-polarization, for a specific choice of $h$ and $\delta$. We have also used the approximate solution $\widehat{u}_{\delta, 1}^{q, h}$ to compute a physical quantity of interest: the so called absorptance $A_{\delta}^{q, h}$ (see for instance [23]).

\subsection{Example 1: Planar backreflector}

The first test allows us to validate the method and its implementation. We have chosen a planar backreflector in which the material occupying $\Omega_{\delta}$ has a uniform relative permittivity $\varepsilon_{r}^{g}(x) \equiv-0.5488+0.1663 \mathrm{i}$. For this problem, for each polarization state $q=s$ or $q=p$, the exact solution $u^{q}(x, z)$ of $(2.7)$ and the corresponding exact absorptance $A^{q}$ can be analytically determined using a textbook approach [2].

For each polarization state, we have computed the relative errors

$$
e_{u^{q}}=\frac{\left(\int_{\widehat{\Omega}_{\gamma}}\left|u^{q}-\widehat{u}_{\delta, 1}^{q, h}\right|^{2} \mathrm{~d} x \mathrm{~d} z\right)^{1 / 2}}{\left(\int_{\widehat{\Omega}_{\gamma}}\left|u^{q}\right|^{2} \mathrm{~d} x \mathrm{~d} z\right)^{1 / 2}} \text { and } e_{A^{q}}=\frac{\left|A^{q}-A_{\delta}^{q, h}\right|}{\left|A^{q}\right|}, \quad q \in\{s, p\} .
$$

We have chosen $\gamma$ large enough $(\gamma=12.5 \mathrm{~nm})$, so that $\widehat{\Omega}_{\gamma}$ and $\Omega_{\delta}$ do not intersect for any value of $\delta$ in our computations.

In order to evaluate the performance of the asymptotic model with respect to the parameter $\delta$, we display in Tables 1 and 2 values of the relative errors $e_{u^{s}}$ and $e_{A^{s}}$, respectively, for varying $\delta$ and $h$.

In spite of the fact that $u^{s}$ and $u^{p}$ are expected to have a similar behavior, the asymptotic models to compute them differ. However the errors $e_{u^{s}}$ and $e_{A^{s}}$ are essentially the same as $e_{u^{p}}$ and $e_{A^{p}}$, respectively. This is the reason why we only report the former in Tables 1 and 2 .

Many of the errors reported in Tables 1 and 2 correspond to values of $\delta$ and $h$ for which the discretization and the asymptotic modeling errors are of a similar size. Because of this, the convergence behavior can be clearly seen only on the last rows and columns of these tables (where the errors arising from the asymptotic modeling or the discretization, respectively, are negligible).

We report in Figure 3 (left) error curves for $e_{u^{s}}$ and $e_{A^{s}}$ versus $\delta$ for a very fine mesh $(h=2.21 \mathrm{~nm})$. These plots show that the errors decrease with order $O\left(\delta^{2}\right)$ as the theory predicts.

Finally, to validate our FEM solver, we display in Figure 3 (right), $e_{u^{s}}$ versus $h$ for an extremely thin grating layer $(\delta=6.103125 \mathrm{e}-03 \mathrm{~nm})$. Standard FEM theory [3] predicts that the rate of convergence of $e_{u^{p}}$ must be of order $O\left(h^{4}\right)$. In Figure 3 (right) we observe exactly this trend, except for the smallest value of $h$ for which the asymptotic modeling error dominates the FEM error. 
TABle 1. Relative error $e_{u^{s}}$ versus $\delta(\mathrm{nm})$ and $h(\mathrm{~nm})$ for Example 1 . The number $N_{e}$ of triangular elements is shown in parentheses for each of the values of $h$ in the table.

\begin{tabular}{lccccc}
\hline \hline & \multicolumn{5}{c}{$h\left(N_{e}\right)$} \\
\cline { 2 - 6 }$\delta(\mathrm{nm})$ & $35.36(224)$ & $17.68(896)$ & $8.84(3584)$ & $4.42(14336)$ & $2.21(57344)$ \\
\hline 12.5 & $5.2675 \mathrm{e}-02$ & $5.2683 \mathrm{e}-02$ & $5.5093 \mathrm{e}-02$ & $5.5093 \mathrm{e}-02$ & $5.5093 \mathrm{e}-02$ \\
6.25 & $1.3077 \mathrm{e}-02$ & $1.3081 \mathrm{e}-02$ & $1.3081 \mathrm{e}-02$ & $1.3381 \mathrm{e}-02$ & $1.3381 \mathrm{e}-02$ \\
3.125 & $3.2539 \mathrm{e}-03$ & $3.2564 \mathrm{e}-03$ & $3.2563 \mathrm{e}-03$ & $3.2563 \mathrm{e}-03$ & $3.2935 \mathrm{e}-03$ \\
1.5625 & $8.1168 \mathrm{e}-04$ & $8.1214 \mathrm{e}-04$ & $8.1207 \mathrm{e}-04$ & $8.1206 \mathrm{e}-04$ & $8.1205 \mathrm{e}-04$ \\
$7.812 \mathrm{e}-01$ & $2.0886 \mathrm{e}-04$ & $2.0258 \mathrm{e}-04$ & $2.0249 \mathrm{e}-04$ & $2.0248 \mathrm{e}-04$ & $2.0248 \mathrm{e}-04$ \\
$3.906 \mathrm{e}-01$ & $7.7185 \mathrm{e}-05$ & $5.0841 \mathrm{e}-05$ & $5.0657 \mathrm{e}-05$ & $5.0650 \mathrm{e}-05$ & $5.0649 \mathrm{e}-05$ \\
$1.953 \mathrm{e}-01$ & $6.1086 \mathrm{e}-05$ & $1.3245 \mathrm{e}-05$ & $1.2667 \mathrm{e}-05$ & $1.2658 \mathrm{e}-05$ & $1.2658 \mathrm{e}-05$ \\
$9.765 \mathrm{e}-02$ & $6.0182 \mathrm{e}-05$ & $4.9215 \mathrm{e}-06$ & $3.1794 \mathrm{e}-06$ & $3.1644 \mathrm{e}-06$ & $3.1638 \mathrm{e}-06$ \\
$4.8825 \mathrm{e}-02$ & $6.0172 \mathrm{e}-05$ & $3.8188 \mathrm{e}-06$ & $8.3173 \mathrm{e}-07$ & $7.9152 \mathrm{e}-07$ & $7.9086 \mathrm{e}-07$ \\
$2.44125 \mathrm{e}-02$ & $6.0176 \mathrm{e}-05$ & $3.7321 \mathrm{e}-06$ & $3.1203 \mathrm{e}-07$ & $1.9875 \mathrm{e}-07$ & $1.9769 \mathrm{e}-07$ \\
$1.220625 \mathrm{e}-02$ & $6.0174 \mathrm{e}-05$ & $3.7244 \mathrm{e}-06$ & $2.4233 \mathrm{e}-07$ & $5.2073 \mathrm{e}-08$ & $4.9411 \mathrm{e}-08$ \\
$6.103125 \mathrm{e}-03$ & $6.0172 \mathrm{e}-05$ & $3.7232 \mathrm{e}-06$ & $2.3649 \mathrm{e}-07$ & $1.9636 \mathrm{e}-08$ & $1.2366 \mathrm{e}-08$ \\
\hline
\end{tabular}

TABLE 2. Relative error $e_{A^{s}}$ versus $\delta(\mathrm{nm})$ and $h(\mathrm{~nm})$ for Example 1 . The number $N_{e}$ of triangular elements is shown in parentheses for each of the values of $h$ in the table.

\begin{tabular}{lccccc}
\hline \hline & \multicolumn{5}{c}{$h\left(N_{e}\right)$} \\
\cline { 2 - 5 }$\delta(\mathrm{nm})$ & $35.36(224)$ & $17.68(896)$ & $8.84(3584)$ & $4.42(14336)$ & $2.21(57344)$ \\
\hline 12.5 & $2.7768 \mathrm{e}-02$ & $2.7778 \mathrm{e}-02$ & $2.7781 \mathrm{e}-02$ & $2.7780 \mathrm{e}-02$ & $2.7780 \mathrm{e}-02$ \\
6.25 & $6.0088 \mathrm{e}-03$ & $6.0007 \mathrm{e}-03$ & $6.0006 \mathrm{e}-03$ & $6.0008 \mathrm{e}-03$ & $6.0008 \mathrm{e}-03$ \\
3.125 & $1.3919 \mathrm{e}-03$ & $1.3754 \mathrm{e}-03$ & $1.3745 \mathrm{e}-03$ & $1.3745 \mathrm{e}-03$ & $1.3745 \mathrm{e}-03$ \\
1.5625 & $3.4925 \mathrm{e}-04$ & $3.2862 \mathrm{e}-04$ & $3.2737 \mathrm{e}-04$ & $3.2731 \mathrm{e}-04$ & $3.2731 \mathrm{e}-04$ \\
$7.812 \mathrm{e}-01$ & $1.0393 \mathrm{e}-04$ & $8.1280 \mathrm{e}-05$ & $7.9835 \mathrm{e}-05$ & $7.9749 \mathrm{e}-05$ & $7.9745 \mathrm{e}-05$ \\
$3.906 \mathrm{e}-01$ & $4.4983 \mathrm{e}-05$ & $2.1316 \mathrm{e}-05$ & $1.9776 \mathrm{e}-05$ & $1.9679 \mathrm{e}-05$ & $1.9674 \mathrm{e}-05$ \\
$1.953 \mathrm{e}-01$ & $3.0754 \mathrm{e}-05$ & $6.5822 \mathrm{e}-06$ & $4.9945 \mathrm{e}-06$ & $4.8920 \mathrm{e}-06$ & $4.8858 \mathrm{e}-06$ \\
$9.765 \mathrm{e}-02$ & $2.7365 \mathrm{e}-05$ & $2.9409 \mathrm{e}-06$ & $1.3295 \mathrm{e}-06$ & $1.2243 \mathrm{e}-06$ & $1.2177 \mathrm{e}-06$ \\
$4.8825 \mathrm{e}-02$ & $2.6591 \mathrm{e}-05$ & $2.0410 \mathrm{e}-06$ & $4.1765 \mathrm{e}-07$ & $3.1104 \mathrm{e}-07$ & $3.0431 \mathrm{e}-07$ \\
$2.44125 \mathrm{e}-02$ & $2.6433 \mathrm{e}-05$ & $1.8198 \mathrm{e}-06$ & $1.9053 \mathrm{e}-07$ & $8.3232 \mathrm{e}-08$ & $7.6419 \mathrm{e}-08$ \\
$1.220625 \mathrm{e}-02$ & $2.6411 \mathrm{e}-05$ & $1.7662 \mathrm{e}-06$ & $1.3400 \mathrm{e}-07$ & $2.6361 \mathrm{e}-08$ & $1.9506 \mathrm{e}-08$ \\
$6.103125 \mathrm{e}-03$ & $2.6414 \mathrm{e}-05$ & $1.7537 \mathrm{e}-06$ & $1.1998 \mathrm{e}-07$ & $1.2163 \mathrm{e}-08$ & $5.2873 \mathrm{e}-09$ \\
\hline
\end{tabular}

\subsection{Example 2: Periodic backreflector with rectangular corrugations}

For the second test, we have considered a backreflector with rectangular corrugations of height $\delta$ and width $L_{1}=200 \mathrm{~nm}$, as shown in Figure 4. In this case, the discontinuous coefficient $\varepsilon_{r}^{g}$ is defined as follows (see Fig. 4):

$$
\varepsilon_{r}^{g}(x)=\left\{\begin{array}{l}
\varepsilon_{r}^{+}, x \in\left(0, \frac{L-L_{1}}{2}\right) \cup\left(\frac{L+L_{1}}{2}, L\right), \\
\varepsilon_{r}^{-}, x \in\left(\frac{L-L_{1}}{2}, \frac{L+L_{1}}{2}\right) .
\end{array}\right.
$$

Let us remark that this kind of corrugation, which is usual in practice, does not satisfy the smoothness assumption on $\varepsilon_{r}^{g}$ used in the theoretical analysis for Theorem 4.5 to hold. Nevertheless, our numerical experiments will show that the proposed strategy works for such a piecewise constant functions $\varepsilon_{r}^{g}$, too.

Since no analytical solution is know for a backreflector like this, we have used as a reference solution the FEM solution of the full model (2.7) computed on a very fine mesh $(h=2.21 \mathrm{~nm})$. We denote this reference solution by $\breve{u}(x, z)$ and by $\breve{A}$ the corresponding absorptance. Using this reference solution, we have computed 

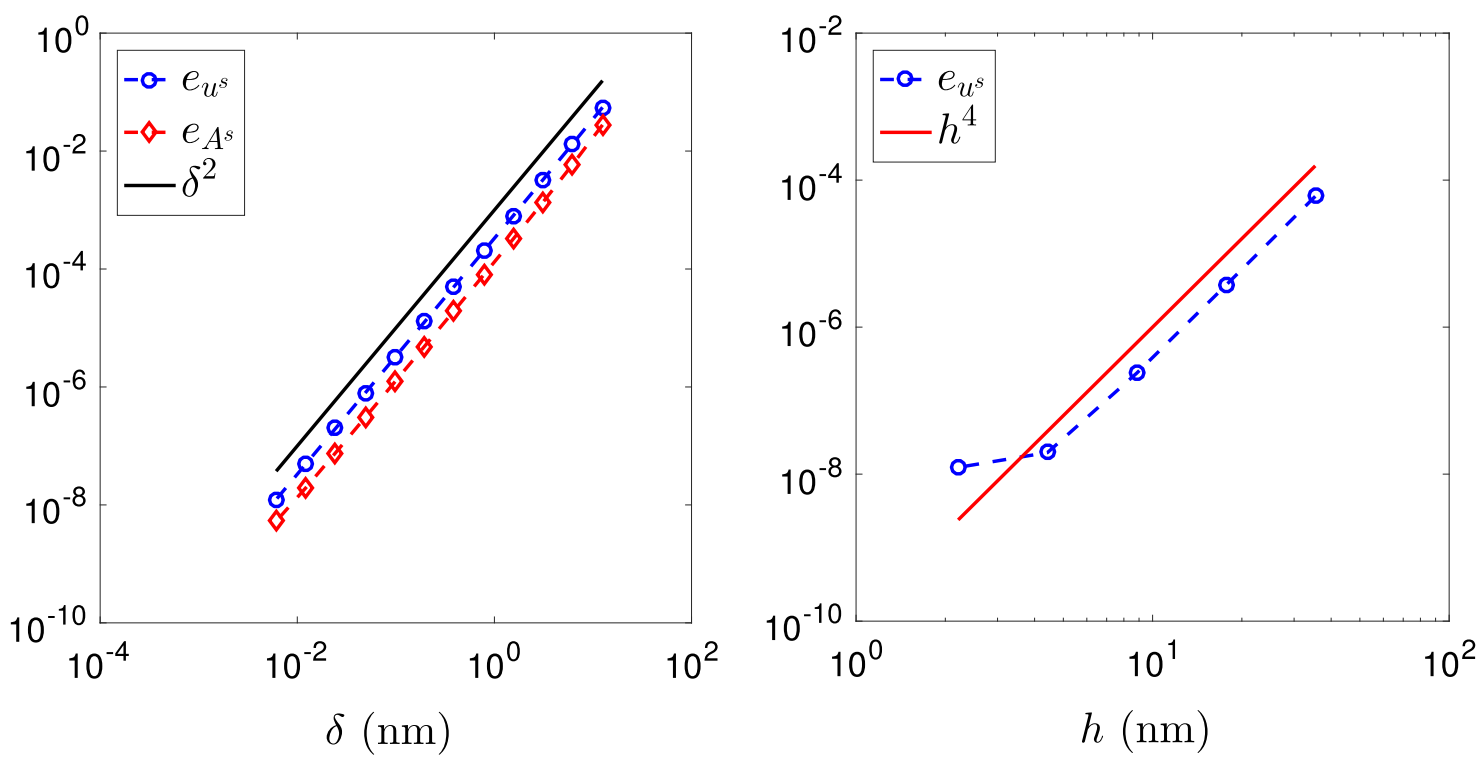

Figure 3. Example 1. Left: computed values of the relative errors $e_{u^{s}}$ (identified by blue $\circ$ ) and $e_{A^{s}}($ red $\diamond)$ versus $\delta$ for $h=2.21 \mathrm{~nm}$; solid black line indicate $\delta^{2}$ dependence. Right: computed values (blue o) of the relative error $e_{u^{s}}$ versus $h$ for $\delta=6.103125 \mathrm{e}-03 \mathrm{~nm}$; solid red line indicate $h^{4}$ dependence.

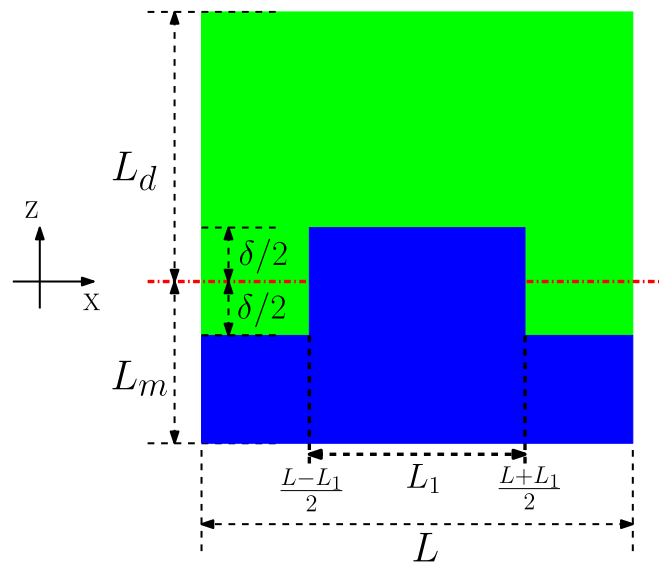

FiguRE 4. Example 2: Domain of a backreflector with a rectangular corrugation.

the relative errors

$$
\breve{e}_{u^{q}}=\frac{\left(\int_{\widehat{\Omega}_{\gamma}}\left|\breve{u}^{q}-\widehat{u}_{\delta, 1}^{q, h}\right|^{2} \mathrm{~d} x \mathrm{~d} z\right)^{1 / 2}}{\left(\int_{\widehat{\Omega}_{\gamma}}\left|\breve{u}^{q}\right|^{2} \mathrm{~d} x \mathrm{~d} z\right)^{1 / 2}} \text { and } \breve{e}_{A^{q}}=\frac{\left|\breve{A}^{q}-A_{\delta}^{q, h}\right|}{\left|\breve{A}^{q}\right|}, \quad q \in\{s, p\},
$$



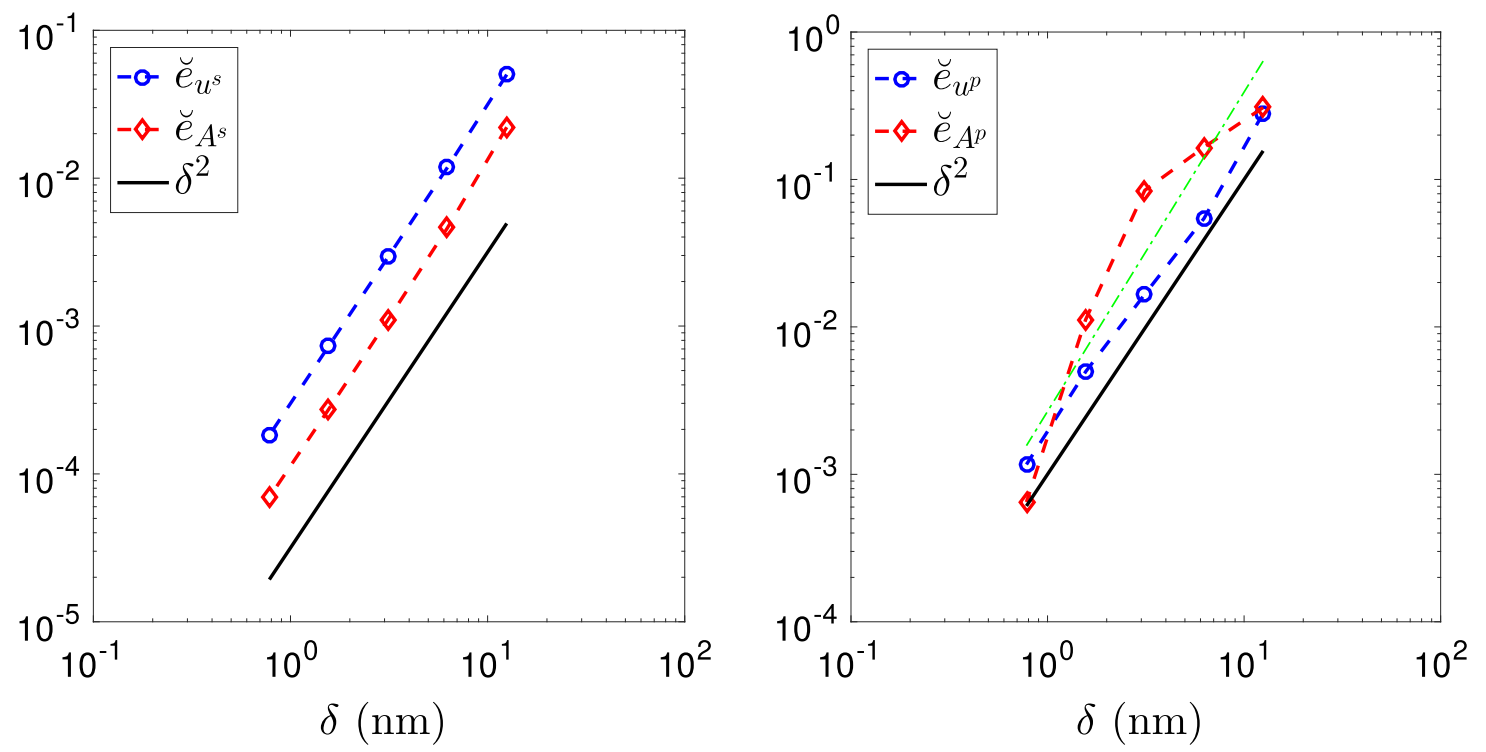

Figure 5. Example 2. Computed values of the relative errors $\breve{e}_{u^{q}}$ (identified by blue $\circ$ ) and $\breve{e}_{A^{q}}($ red $\diamond)$ versus $\delta$ for $h=2.21 \mathrm{~nm}$. Solid black lines indicate $\delta^{2}$ dependence. Left: $q=s$. Right: $q=p$; the dash-dotted green line corresponds to a least squares fitting of $\breve{e}_{A^{p}}$.

for different values of $\delta$. Notice that since the exact solution depends on $\delta$, the reference solution had to be computed for each value of this parameter. Let us remark that this FEM reference solution has been validated in [26] by comparing it with an RCWA solution. In fact, it has been reported in that reference that the FEM and RCWA solutions agree within $3 \%$ in absorptances and within $5 \%$ in $L^{2}(\Omega)$.

Figure 5 shows error curves for $\breve{e}_{u^{q}}$ and $\breve{e}_{A^{q}}$ versus $\delta$ for $q=s$ (left) and $q=p$ (right). These plots show that the errors $\breve{e}_{u^{s}}$, $\breve{e}_{A^{s}}$ and $\breve{e}_{u^{p}}$ decrease with order $O\left(\delta^{2}\right)$. Instead, the order of convergence of $\breve{e}_{A^{p}}$ is not clear. However, a least squares fitting of these errors decreases with order around $O\left(\delta^{2}\right)$, as can be seen in the same figure.

According to [26], the solution $\breve{u}^{q}(x, z)$ of the full model contains strong singularities near metallic corners, due to the type of partial differential equation involved. Hence, in principle, any numerical approximation of the actual solution is not very accurate, unless the mesh is sufficiently refined in the proximity of these corners. In practice, this implies dealing with extremely fine meshes and, hence, expensive solutions in terms of computer cost. This is a classical problem in grating theory [16,17], specially for $p$ polarization. This issue affects the numerical solution of the full model [26] as well as those obtained by other approaches as, for instance, the RCWA method [28] and could affect the asymptotic model as well. However, a clear advantage of the proposed asymptotic approach is that this kind of overrefined meshes are no longer needed.

\section{Appendix A.}

The main goal of this section is to derive the transmission conditions (3.9), as well as expressions (3.12) for the near field terms within the grating.

\section{A.1. Equations for the first term of the asymptotic expansions}

For $n=0$, according to Proposition 3.4, outside the grating layer we have

$$
U_{0}(x, \xi)=u_{0}^{ \pm}(x, 0), \quad x \in(0, L), \quad \frac{1}{2} \leq|\xi| \leq 2 .
$$


Into the grating, since $B(x, \delta \xi)=B^{g}(x)$ does not depend on $\xi$, the first equation in (3.5) reads

$$
\frac{\partial}{\partial \xi}\left(B^{g}(x) \frac{\partial U_{0}(x, \xi)}{\partial \xi}\right)=0, \quad x \in(0, L), \quad|\xi| \leq \frac{1}{2}
$$

Then, there exist functions $q_{0}(x)$ and $r_{0}(x)$ such that

$$
U_{0}(x, \xi)=q_{0}(x)+\frac{r_{0}(x)}{B^{g}(x)} \xi, \quad x \in(0, L), \quad|\xi| \leq \frac{1}{2} .
$$

Since $U_{0}$ is continuous on $\xi= \pm \frac{1}{2}$, we have that

$$
\begin{aligned}
& U_{0}\left(x, \frac{1}{2}^{-}\right)=U_{0}\left(x, \frac{1}{2}^{+}\right) \Longrightarrow q_{0}(x)+\frac{1}{2} \frac{r_{0}(x)}{B^{g}(x)}=u_{0}^{+}(x, 0), \\
& U_{0}\left(x,-\frac{1}{2}^{+}\right)=U_{0}\left(x,-\frac{1}{2}^{-}\right) \Longrightarrow q_{0}(x)-\frac{1}{2} \frac{r_{0}(x)}{B^{g}(x)}=u_{0}^{-}(x, 0),
\end{aligned}
$$

while, from the continuity of $B \frac{\partial U_{0}}{\partial \xi}$,

$$
\begin{aligned}
& B^{g}(x) \frac{\partial U_{0}}{\partial \xi}\left(x, \frac{1}{2}^{-}\right)=B^{+} \frac{\partial U_{0}}{\partial \xi}\left(x, \frac{1}{2}^{+}\right) \Longrightarrow r_{0}(x)=0 \\
& B^{g}(x) \frac{\partial U_{0}}{\partial \xi}\left(x,-\frac{1}{2}^{+}\right)=B^{-} \frac{\partial U_{0}}{\partial \xi}\left(x,-\frac{1}{2}^{-}\right) \Longrightarrow r_{0}(x)=0 .
\end{aligned}
$$

Therefore, $r_{0}(x)$ vanishes and $u_{0}^{+}(x, 0)=q_{0}(x)=u_{0}^{-}(x, 0)$. Hence, (A.1) and (A.2) imply that

$$
U_{0}(x, \xi)=u_{0}^{+}(x, 0)=u_{0}^{-}(x, 0), \quad x \in(0, L), \quad|\xi| \leq 2 .
$$

In particular, $U_{0}$ does not depend on $\xi$. Moreover, this implies that $u_{0}$ is continuous across $\Gamma$, so that

$$
\left[u_{0}\right](x)=u_{0}^{+}(x, 0)-u_{0}^{-}(x, 0)=0, \quad x \in(0, L),
$$

and

$$
\left\langle u_{0}\right\rangle(x)=\frac{u_{0}^{+}(x, 0)+u_{0}^{-}(x, 0)}{2}=U_{0}(x), \quad x \in(0, L) .
$$

Note that since $U_{0}$ does not depend on $\xi$, here and in what follows we make the abuse of language of writing $U_{0}(x)$ instead of $U_{0}(x, \xi)$.

\section{A.2. Equations for the second term of the asymptotic expansions}

For $n=1$, according to Proposition 3.4, outside the grating layer we have

$$
U_{1}(x, \xi)=u_{1}^{ \pm}(x, 0)+\xi \frac{\partial u_{0}^{ \pm}}{\partial z}(x, 0), \quad x \in(0, L), \quad \frac{1}{2} \leq|\xi| \leq 2,
$$

while, into the grating, the first equation in (3.5) reads

$$
\frac{\partial}{\partial \xi}\left(B^{g}(x) \frac{\partial U_{1}(x, \xi)}{\partial \xi}\right)=0, \quad x \in(0, L), \quad|\xi| \leq \frac{1}{2} .
$$

Then, there exist functions $q_{1}(x)$ and $r_{1}(x)$ such that

$$
U_{1}(x, \xi)=q_{1}(x)+\frac{r_{1}(x)}{B^{g}(x)} \xi, \quad x \in(0, L), \quad|\xi| \leq \frac{1}{2} .
$$


Proceeding as in the previous step, from the continuity of $U_{1}$ and $B \frac{\partial U_{1}}{\partial \xi}$ on $\xi= \pm \frac{1}{2}$ we obtain

$$
\begin{aligned}
u_{1}^{ \pm}(x, 0) \pm \frac{1}{2} \frac{\partial u_{0}^{ \pm}}{\partial z}(x, 0) & =q_{1}(x) \pm \frac{1}{2} \frac{r_{1}(x)}{B^{g}(x)}, \\
B^{+} \frac{\partial u_{0}^{+}}{\partial z}(x, 0) & =r_{1}(x)=B^{-} \frac{\partial u_{0}^{-}}{\partial z}(x, 0) .
\end{aligned}
$$

From the latter, we derive

$$
\left[B \frac{\partial u_{0}}{\partial z}\right](x)=0
$$

and

$$
\left\langle B \frac{\partial u_{0}}{\partial z}\right\rangle(x)=r_{1}(x) .
$$

For the jump of $u_{1}$ across $\Gamma$, by subtracting both expressions in (A.6) we obtain

$$
\left[u_{1}\right](x)=-\frac{1}{2} \frac{\partial u_{0}^{-}}{\partial z}(x, 0)-\frac{1}{2} \frac{\partial u_{0}^{+}}{\partial z}(x, 0)+\frac{r_{1}(x)}{B^{g}(x)}=-\frac{1}{2 B^{-}} r_{1}(x)-\frac{1}{2 B^{+}} r_{1}(x)+\frac{r_{1}(x)}{B^{g}(x)},
$$

where we have used (A.7) for the last equality. Then, (A.8) leads to

$$
\left[u_{1}\right](x)=\left(\frac{1}{B^{g}(x)}-\left\langle\frac{1}{B}\right\rangle\right)\left\langle B \frac{\partial u_{0}}{\partial z}\right\rangle(x), \quad x \in(0, L) .
$$

Finally, to obtain from (A.5) an expression for $U_{1}$ into the grating, we need expressions of $q_{1}$ and $r_{1}$. For the latter, we already have (A.8). For the former, we average both equations in (A.6) and obtain

$$
q_{1}(x)=\left\langle u_{1}\right\rangle(x)+\frac{1}{4} \frac{\partial u_{0}^{+}}{\partial z}(x, 0)-\frac{1}{4} \frac{\partial u_{0}^{-}}{\partial z}(x, 0)=\left\langle u_{1}\right\rangle(x)+\frac{1}{4 B^{+}} r_{1}(x)-\frac{1}{4 B^{-}} r_{1}(x),
$$

where we have used (A.7) for the last equality. Then, substituting this into (A.5) and using (A.8) to eliminate $r_{1}(x)$ leads to

$$
U_{1}(x, \xi)=\left\langle u_{1}\right\rangle(x)+\left(\frac{\xi}{B^{g}(x)}+\frac{1}{4}\left[\frac{1}{B}\right]\right)\left\langle B \frac{\partial u_{0}}{\partial z}\right\rangle(x), \quad x \in(0, L), \quad|\xi| \leq \frac{1}{2} .
$$

\section{A.3. Equations for the third term of the asymptotic expansions}

For $n=2$, according to Proposition 3.4, outside the grating layer we have

$$
U_{2}(x, \xi)=u_{2}^{ \pm}(x, 0)+\xi \frac{\partial u_{1}^{ \pm}}{\partial z}(x, 0)+\frac{\xi^{2}}{2} \frac{\partial^{2} u_{0}^{ \pm}}{\partial z^{2}}(x, 0), \quad x \in(0, L), \quad \frac{1}{2} \leq|\xi| \leq 2 .
$$

Into the grating, since $B(x, \delta \xi)=B^{g}(x), b(x, \delta \xi)=b^{g}(x)$ and $U_{0}(x)$ do not depend on $\xi$, the first equation in (3.5) reads

$$
\frac{\partial}{\partial \xi}\left(B^{g}(x) \frac{\partial U_{2}(x, \xi)}{\partial \xi}\right)=-\left(\frac{\partial}{\partial x}\left(B^{g}(x) \frac{\partial U_{0}(x)}{\partial x}\right)+k_{0}^{2} b^{g}(x) U_{0}(x)\right), \quad x \in(0, L), \quad|\xi| \leq \frac{1}{2}
$$

and, hence, there exist functions $q_{2}(x)$ and $r_{2}(x)$ such that

$$
U_{2}(x, \xi)=q_{2}(x)+\frac{r_{2}(x)}{B^{g}(x)} \xi-\frac{1}{2 B^{g}(x)}\left(\frac{\partial}{\partial x}\left(B^{g}(x) \frac{\partial U_{0}(x)}{\partial x}\right)+k_{0}^{2} b^{g}(x) U_{0}(x)\right) \xi^{2}, \quad x \in(0, L), \quad|\xi| \leq \frac{1}{2} .
$$


Since $U_{2}$ is continuous on $\xi= \pm \frac{1}{2}$, by equating (A.10) and (A.11) at those values of $\xi$, we obtain

$$
u_{2}^{ \pm}(x, 0) \pm \frac{1}{2} \frac{\partial u_{1}^{ \pm}}{\partial z}(x, 0)+\frac{1}{8} \frac{\partial^{2} u_{0}^{ \pm}}{\partial z^{2}}(x, 0)=q_{2}(x) \pm \frac{r_{2}(x)}{2 B^{g}(x)}-\frac{1}{8 B^{g}(x)}\left(\frac{\partial}{\partial x}\left(B^{g}(x) \frac{\partial U_{0}(x)}{\partial x}\right)+k_{0}^{2} b^{g}(x) U_{0}(x)\right) .
$$

Then, averaging and subtracting both equations above, we obtain

$$
\begin{aligned}
q_{2}(x)= & \frac{1}{2} u_{2}^{+}(x, 0)+\frac{1}{2} u_{2}^{-}(x, 0)+\frac{1}{4} \frac{\partial u_{1}^{+}}{\partial z}(x, 0)-\frac{1}{4} \frac{\partial u_{1}^{-}}{\partial z}(x, 0)+\frac{1}{16} \frac{\partial^{2} u_{0}^{+}}{\partial z^{2}}(x, 0)+\frac{1}{16} \frac{\partial^{2} u_{0}^{-}}{\partial z^{2}}(x, 0) \\
& +\frac{1}{8 B^{g}(x)}\left(\frac{\partial}{\partial x}\left(B^{g}(x) \frac{\partial U_{0}(x)}{\partial x}\right)+k_{0}^{2} b^{g}(x) U_{0}(x)\right)
\end{aligned}
$$

and

$$
r_{2}(x)=B^{g}(x)\left(u_{2}^{+}(x, 0)-u_{2}^{-}(x, 0)+\frac{1}{2} \frac{\partial u_{1}^{+}}{\partial z}(x, 0)+\frac{1}{2} \frac{\partial u_{1}^{-}}{\partial z}(x, 0)+\frac{1}{8} \frac{\partial^{2} u_{0}^{+}}{\partial z^{2}}(x, 0)-\frac{1}{8} \frac{\partial^{2} u_{0}^{-}}{\partial z^{2}}(x, 0)\right) .
$$

Note that both functions, $q_{2}(x)$ and $r_{2}(x)$, are infinitely smooth in $[0, L]$.

On the other hand, since $B \frac{\partial U_{2}}{\partial \xi}$ is also continuous on $\xi= \pm \frac{1}{2}$, differentiating (A.10) and (A.11) with respect to $\xi$ and evaluating at those values of $\xi$ lead to

$$
B^{ \pm} \frac{\partial u_{1}^{ \pm}}{\partial z}(x, 0) \pm B^{ \pm} \frac{\partial^{2} u_{0}^{ \pm}}{\partial z^{2}}(x, 0)=\mp \frac{1}{2}\left(\frac{\partial}{\partial x}\left(B^{g}(x) \frac{\partial U_{0}(x)}{\partial x}\right)+k_{0}^{2} b^{g}(x) U_{0}(x)\right)+r_{2}(x) .
$$

For the jump of $B \frac{\partial u_{1}}{\partial z}$ across $\Gamma$, we subtract the equations above and obtain

$$
\left[B \frac{\partial u_{1}}{\partial z}\right](x)=-\left(\frac{\partial}{\partial x}\left(B^{g}(x) \frac{\partial U_{0}(x)}{\partial x}\right)+k_{0}^{2} b^{g}(x) U_{0}(x)\right)-B^{+} \frac{\partial^{2} u_{0}^{+}}{\partial z^{2}}(x, 0)-B^{-} \frac{\partial^{2} u_{0}^{-}}{\partial z^{2}}(x, 0), \quad x \in(0, L) .
$$

However, this equation involves the undetermined quantities $\frac{\partial^{2} u_{0}^{ \pm}}{\partial z^{2}}(x, 0)$. To eliminate them, we resort again to the first equation in (3.5), now for $|\xi| \geq \frac{1}{2}$. Since in such a case, $B(x, \delta \xi)=B^{ \pm}$and $b(x, \delta \xi)=b^{ \pm}$, the equation reads

$$
\frac{\partial}{\partial \xi}\left(B^{ \pm} \frac{\partial U_{2}(x, \xi)}{\partial \xi}\right)=-\left(\frac{\partial}{\partial x}\left(B^{ \pm} \frac{\partial U_{0}(x)}{\partial x}\right)+k_{0}^{2} b^{ \pm} U_{0}(x)\right), \quad x \in(0, L), \quad \frac{1}{2} \leq|\xi| \leq 2 .
$$

Hence, as above, there exist functions $q_{2}^{ \pm}(x)$ and $r_{2}^{ \pm}(x)$ such that

$$
U_{2}(x, \xi)=q_{2}^{ \pm}(x)+\frac{r_{2}^{ \pm}(x)}{B^{ \pm}} \xi-\frac{1}{2 B^{ \pm}}\left(\frac{\partial}{\partial x}\left(B^{ \pm} \frac{\partial U_{0}(x)}{\partial x}\right)+k_{0}^{2} b^{ \pm} U_{0}(x)\right) \xi^{2}, \quad x \in(0, L), \quad \frac{1}{2} \leq|\xi| \leq 2 .
$$

Since (A.10) holds true in the same domain, identifying both expressions we derive that

$$
u_{2}^{ \pm}(x, 0)=q_{2}^{ \pm}(x), \quad \frac{\partial u_{1}^{ \pm}}{\partial z}(x, 0)=\frac{r_{2}^{ \pm}(x)}{B^{ \pm}} \quad \text { and } \quad \frac{1}{2} \frac{\partial^{2} u_{0}^{ \pm}}{\partial z^{2}}(x, 0)=-\frac{1}{2 B^{ \pm}}\left(\frac{\partial}{\partial x}\left(B^{ \pm} \frac{\partial U_{0}(x)}{\partial x}\right)+k_{0}^{2} b^{ \pm} U_{0}(x)\right) .
$$

Then, using the last equation and (A.4) in (A.14), we obtain

$$
\left[B \frac{\partial u_{1}}{\partial z}\right](x)=-\frac{\partial}{\partial x}\left(\left(B^{g}(x)-\langle B\rangle\right) \frac{\partial\left\langle u_{0}\right\rangle}{\partial x}(x)\right)-k_{0}^{2}\left(b^{g}(x)-\langle b\rangle\right)\left\langle u_{0}\right\rangle(x), \quad x \in(0, L) .
$$

The equations derived above that appear within boxes have been claimed to hold without a proof in Section 3 . In fact, they correspond either to the transmission conditions (3.9) or to equations (3.12) for the near field in the grating. To end this paper, we use some of the equations derived above to prove the following result, which has been used in the proof of Lemma 4.4. 
Lemma A.1. $U_{0}^{\delta}, U_{1}^{\delta}$ and $U_{2}^{\delta}$ are infinitely differentiable with respect to $x$ in $O_{\delta}$ and their $x$-derivatives are uniformly bounded independently of $\delta$.

Proof. Out of the grating, there is nothing to prove. In fact, according to Assumption $3.2, U_{n}(x, \xi)$ are infinitely smooth for $\frac{1}{2} \leq|\xi| \leq 2$ and, then, $U_{n}^{\delta}(x, z)$ are infinitely smooth for $\frac{\delta}{2} \leq|z| \leq 2 \delta$.

Into the grating $\left(|z| \leq \frac{\delta}{2}\right.$ ), we must recall the assumed smoothness of $\varepsilon_{r}^{g}(x)$ and $u_{n}^{ \pm}$(cf. Assumption 3.1 for the latter). Then, the property for $U_{0}^{\delta}$ holds immediately because of (A.3). For $U_{1}^{\delta}$, it follows from (A.9). Finally, for $U_{2}^{\delta}$ it is derived from (A.11) to (A.13) and the already proved smoothness of $U_{0}(x)$.

Acknowledgements. Research partially supported by CONICYT-Chile through Becas-Chile program, grant AFB170001 of the PIA Program, "Concurso Apoyo a Centros Científicos y Tecnológicos de Excelencia con Financiamiento Basal", and grants FONDECYT-1160320 and FONDECYT-1200569. The research of P.M. was supported in part by grants from NSF (DMS-1619904) and AFOSR (FA9550-20-1-0024).

\section{REFERENCES}

[1] A. Barnett and L. Greengard, A new integral representation for quasi-periodic scattering problems in two dimensions. BIT $\mathbf{5 1}$ (2011) 67-90

[2] M. Born and E. Wolf, Principles of Optics, 6th edition. Cambridge University (1980).

[3] S.C. Brenner and L.R. Scott, The Mathematical Theory of Finite Element Methods, 2nd edition. Springer (2002).

[4] N. Chateau and J.-P. Hugonin, Algorithm for the rigorous coupled-wave analysis of grating diffraction. J. Opt. Soc. Am. A 11 (1994) 1321-1331.

[5] Z. Chen and $\mathrm{H}$. Wu, An adaptive finite element method with perfectly matched absorbing layers for the wave scattering by periodic structures. SIAM J. Numer. Anal. 41 (2004) 799-826.

[6] B.J. Civiletti, A. Lakhtakia and P.B. Monk, Analysis of the rigorous coupled wave approach for s-polarized light in gratings. J. Comput. Appl. Math. 368 (2019) 112478.

[7] B. Delourme, High-order asymptotics for the electromagnetic scattering by thin periodic layers. Math. Methods Appl. Sci. 38 (2015) 811-833.

[8] B. Delourme, H. Haddar and P. Joly, Approximate models for wave propagation across thin periodic interfaces. J. Math. Pures Appl. 98 (2012) 28-71.

[9] B. Delourme, H. Haddar and P. Joly, On the well-posedness, stability and accuracy of an asymptotic model for thin periodic interfaces in electromagnetic scattering problems. Math. Models Methods Appl. Sci. 23 (2013) 2433-2464.

[10] J. Elschner and G. Schmidt, Diffraction in periodic structures and optimal design of binary gratings. Part I: direct problems and gradient formulas. Math. Meth. Appl. Sci. 21 (1998) 1297-1342.

[11] A. Gillman and A. Barnett, A fast direct solver for quasi-periodic scattering problems. J. Comp. Phys. 248 (2013) $309-322$.

[12] G. Granet and B. Guizal, Efficient implementation of the coupled-wave method for metallic lamellar gratings in TM polarization. J. Opt. Soc. Am. A, 13 (1996) 1019-1023.

[13] H. Haddar, P. Joly and H.-M. Nguyen, Generalized impedance boundary conditions for scattering by strongly absorbing obstacles: the scalar case. Math. Models Methods Appl. Sci. 15 (2005) 1273-1300.

[14] P. Lalanne and G.M. Morris, Highly improved convergence of the coupled-wave method for TM polarization. J. Opt. Soc. Am. A 13 (1996) 779-784.

[15] L. Li, Use of Fourier series in the analysis of discontinuous periodic structures. J. Opt. Soc. Am. A 13 (1996) $1870-1876$.

[16] L. Li, New formulation of the Fourier modal method for crossed surface-relief gratings. J. Opt. Soc. Am. A 14 (1997) $2758-2767$.

[17] E.G. Loewen and E. Popov, Diffraction Gratings and Applications. Marcel Dekker (1997).

[18] D. Maystre, editor. Selected Papers on Diffraction Gratings. SPIE Press (1993).

[19] A. Maurel, J.-J. Marigo and A. Ourir, Homogenization of ultrathin metallo-dielectric structures leading to transmission conditions at an equivalent interface. J. Opt. Soc. Am. B. 33 (2016) 947-956.

[20] M.G. Moharam, E.B. Grann and D.A. Pommet, Formulation for stable and efficient implementation of the rigorous coupledwave analysis of binary gratings. J. Opt. Soc. Am. A 12 (1995) 1068-1076.

[21] P.B. Monk, Finite Element Methods for Maxwell's Equations. Oxford University Press (2003).

[22] Ö. Özdemir, H. Haddar and A. Yaka, Reconstruction of the electromagnetic field in layered media using the concept of approximate transmission conditions. IEEE Trans. Antennas Propag. 59 (2011) 2964-2972.

[23] C. Rivas, M. E. Solano, R. Rodriguez, P. B. Monk and A. Lakhtakia, Asymptotic model for finite-element calculations of diffraction by shallow metallic surface-relief gratings. J. Opt. Soc. Am. A, 34 (2017) 68-79.

[24] M.E. Solano, M. Faryad, A.S. Hall, T.E. Mallouk, P.B. Monk and A. Lakhtakia, Optimization of the absorption efficiency of an amorphous-silicon thin-film tandem solar cell backed by a metallic surface-relief grating, Appl. Opt. 52 (2013) 966-979.

[25] M.E. Solano, M. Faryad, P.B. Monk, T.E. Mallouk and A. Lakhtakia, Periodically multilayered planar optical concentrator for photovoltaic solar cells. Appl. Phys. Lett. 103 (2013) 191115. 
[26] M.E. Solano, M. Faryad, A. Lakhtakia and P.B. Monk, Comparison of rigorous coupled-wave approach and finite element method for photovoltaic devices with periodically corrugated metallic back reflector. J. Opt. Soc. Am. A 31 (2014) $2275-2284$.

[27] M.E. Solano, G. D. Barber, A. Lakhtakia, M. Faryad, P.B. Monk and T.E. Mallouk, Buffer layer between a planar optical concentrator and a solar cell, AIP Adv. 5 (2015) 097150.

[28] M.V. Shuba, M. Faryad, M.E. Solano, P.B. Monk and A. Lakhtakia, Adequacy of the rigorous coupled-wave approach for thinfilm silicon solar cells with periodically corrugated metallic backreflectors: spectral analysis. J. Opt. Soc. Am. A 32 (2015) $1222-1230$.

[29] A. Taflove and S.C. Hagness, Computational Electrodynamics: The Finite-Difference Time-Domain Method, 3rd edition. Artech House (2005). 